\title{
Chapter 4 \\ QCD: The Theory of Strong Interactions
}

\author{
Guido Altarelli and Stefano Forte
}

\subsection{Introduction}

This Chapter ${ }^{1}$ is devoted to a concise introduction to Quantum Chromo-Dynamics (QCD), the theory of strong interactions [1-3]. We start with a general introduction where a broad overview of the strong interactions is presented. The basic principles and the main applications of perturbative QCD will be discussed first (for reviews of the subject, see, for example, [4-6]). Then the methods of non perturbative QCD will be introduced, first the analytic approaches and then the simulations of the theory on a discrete space-time lattice.The main emphasis will be on ideas with a minimum of technicalities.

As discussed in Chap. 2 the QCD theory of strong interactions is an unbroken gauge theory based on the group $S U$ (3) of colour. The eight massless gauge bosons are the gluons $g_{\mu}^{A}$ and matter fields are colour triplets of quarks $q_{i}^{a}$ (in different flavours $i$ ). Quarks and gluons are the only fundamental fields of the Standard Model (SM) with strong interactions (hadrons). As discussed in Chap. 2, the statement that

The author "G. Altarelli" is deceased at the time of publication.

${ }^{1}$ See Chap. 2 for a general introduction to Chaps. 2-4 with updated references.

G. Altarelli

University of Rome 3, Rome, Italy

S. Forte $(\bowtie)$

Dipartimento di Fisica, Università di Milano, Milano, Italy

(C) The Author(s) 2020

H. Schopper (ed.), Particle Physics Reference Library,

https://doi.org/10.1007/978-3-030-38207-0_4 
QCD is a renormalisable gauge theory based on the group $S U(3)$ with colour triplet quark matter fields [7] fixes the QCD lagrangian density to be:

$$
\mathcal{L}=-\frac{1}{4} \sum_{A=1}^{8} F^{A \mu \nu} F_{\mu \nu}^{A}+\sum_{j=1}^{n_{f}} \bar{q}_{j}\left(i \not D-m_{j}\right) q_{j}
$$

Here: $q_{j}$ are the quark fields (of $n_{f}$ different flavours) with mass $m_{j} ; D=D_{\mu} \gamma^{\mu}$, where $\gamma^{\mu}$ are the Dirac matrices and $D_{\mu}$ is the covariant derivative:

$$
D_{\mu}=\partial_{\mu}+i e_{s} \mathbf{g}_{\mu}
$$

$e_{s}$ is the gauge coupling, later we will mostly use, in analogy with QED

$$
\alpha_{s}=\frac{e_{s}^{2}}{4 \pi}
$$

$\mathbf{g}_{\mu}=\sum_{A} t^{A} g_{\mu}^{A}$ where $g_{\mu}^{A}, A=1,8$, are the gluon fields and $t^{A}$ are the $S U(3)$ group generators in the triplet representation of quarks (i.e. $t_{A}$ are $3 \times 3$ matrices acting on $q$ ); the generators obey the commutation relations $\left[t^{A}, t^{B}\right]=i C_{A B C} t^{C}$ where $C_{A B C}$ are the complete antisymmetric structure constants of $S U(3)$ (the normalisation of $C_{A B C}$ and of $e_{s}$ is specified by $\operatorname{Tr}\left[t^{A} t^{B}\right]=\delta^{A B} / 2$ );

$$
F_{\mu \nu}^{A}=\partial_{\mu} g_{\nu}^{A}-\partial_{\nu} g_{\mu}^{A}-e_{s} C_{A B C} g_{\mu}^{B} g_{\nu}^{C}
$$

For quantisation the classical Lagrangian in Eq. (4.1) must be enlarged to contain gauge fixing and ghost terms, as described in Chap.2. The Feynman rules of QCD are listed in Fig. 4.1. The physical vertices in QCD include the gluon-quarkantiquark vertex, analogous to the QED photon-fermion-antifermion coupling, but also the 3-gluon and 4-gluon vertices, of order $e_{s}$ and $e_{s}^{2}$ respectively, which have no analogue in an abelian theory like QED.

The QCD lagrangian in Eq. (4.1) has a simple structure but a very rich dynamical content. It gives rise to a complex spectrum of hadrons, it implies the striking properties of confinement and asymptotic freedom, is endowed with an approximate chiral symmetry which is spontaneously broken, has a highly non trivial topological vacuum structure (instantons, $U(1)_{A}$ symmetry breaking, strong $\mathrm{CP}$ violation (which is a problematic item in QCD possibly connected with new physics, like axions), ...), an intriguing phase transition diagram (colour deconfinement, quarkgluon plasma, chiral symmetry restoration, colour superconductivity, ... ).

Confinement is the property that no isolated coloured charge can exist but only colour singlet particles. For example, the potential between a quark and an antiquark has been studied on the lattice. It has a Coulomb part at short distances and a linearly rising term at long distances:

$$
V_{q \bar{q}} \approx C_{F}\left[\frac{\alpha_{s}(r)}{r}+\ldots+\sigma r\right]
$$




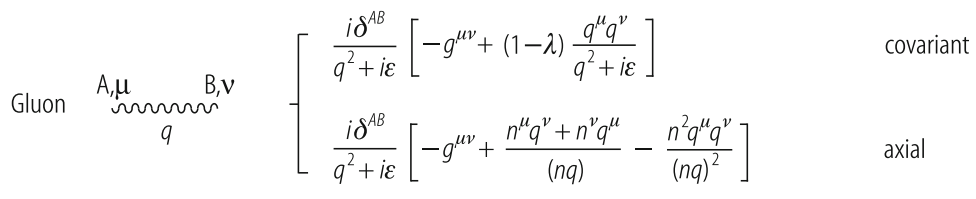

Quark $\frac{i}{p} \quad \frac{i}{\hat{p}-m+i \varepsilon}$

Ghost $\quad \stackrel{P}{p} \quad \frac{i \delta^{A B}}{p^{2}+i \varepsilon} \quad$ covariant
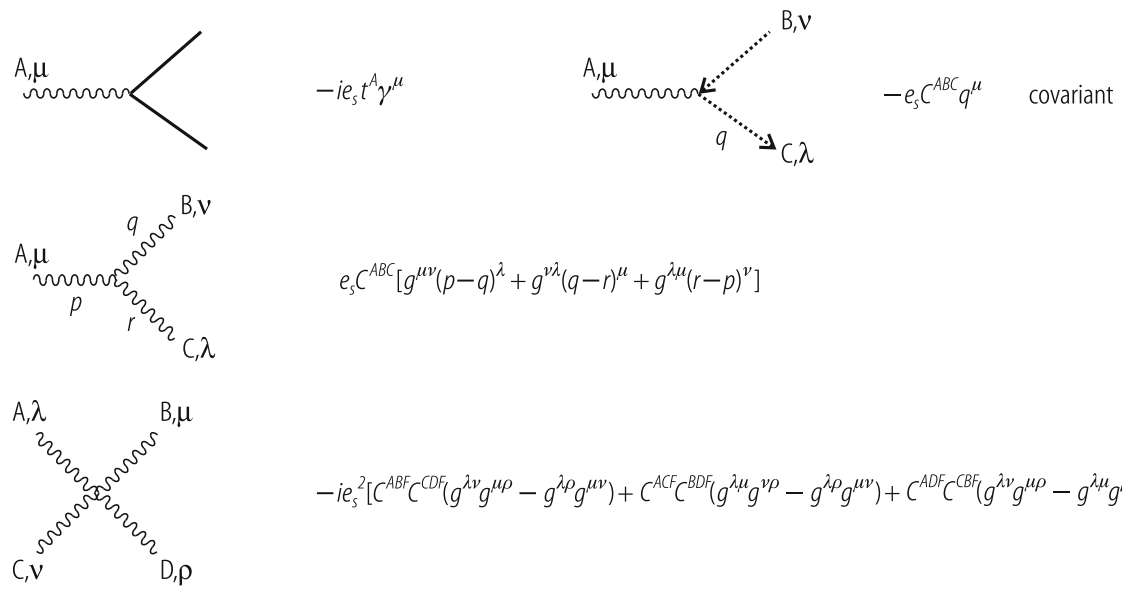

$$
-i e_{s}^{2}\left[C^{A B F} C^{C D F}\left(g^{\lambda v} g^{\mu \rho}-g^{\lambda \rho} g^{\mu \nu}\right)+C^{A C F} C^{B D F}\left(g^{\lambda \mu} g^{\nu \rho}-g^{\lambda \rho} g^{\mu \nu}\right)+C^{A D F} C^{B B F}\left(g^{\lambda \nu} g^{\mu \rho}-g^{\lambda \mu} g^{\rho \nu}\right)\right]
$$

Fig. 4.1 Feynman rules for QCD. The solid lines represent the fermions, the curly lines the gluons, and the dotted lines represent the ghosts (see Chap. 2). The gauge parameter is denoted by $\lambda$. The 3 -gluon vertex is written as if all gluon lines are outgoing

where

$$
C_{F}=\frac{1}{N_{C}} \sum_{A} t^{A} t^{A}=\frac{N_{C}^{2}-1}{2 N_{C}}
$$

with $N_{C}$ the number of colours $\left(N_{C}=3\right.$ in QCD). The scale dependence of $\alpha_{s}$ (the distance $\mathrm{r}$ is Fourier-conjugate to momentum transfer) will be explained in detail in the following. The understanding of the confinement mechanism has much improved thanks to lattice simulations of QCD at finite temperatures and densities. The slope decreases with increasing temperature until it vanishes at a critical temperature $T_{C}$. Above $T_{C}$ the slope remains zero. The phase transitions of colour deconfinement and of chiral restauration appear to happen together on the lattice. A rapid transition is observed in lattice simulations where the energy density $\epsilon(T)$ is seen to sharply increase near the critical temperature for deconfinement and chiral restauration. The critical parameters and the nature of the phase transition 
depend on the number of quark flavours $n_{f}$ and on their masses. For example, for $n_{f}=2$ or $2+1$ (i.e. two light $\mathrm{u}$ and d quarks and one heavier s quark), $T_{C} \sim 175 \mathrm{MeV}$ and $\epsilon\left(T_{C}\right) \sim 0.5-1.0 \mathrm{GeV} / \mathrm{fm}^{3}$. For realistic values of the masses $m_{s}$ and $m_{u, d}$ the phase transition appears to be a second order one, while it becomes first order for very small or very large $m_{u, d, s}$. The hadronic phase and the deconfined phase are separated by a crossover line at small densities and by a critical line at high densities. Determining the exact location of the critical point in $\mathrm{T}$ and $\mu_{B}$ is an important challenge for theory which is also important for the interpretation of heavy ion collision experiments. At high densities the colour superconducting phase is also present with bosonic diquarks acting as Cooper pairs.

A large investment is being done in experiments of heavy ion collisions with the aim of finding some evidence of the quark gluon plasma phase. Many exciting results have been found at the CERN SPS in the past years and more recently at RHIC. The status of the experimental search for the quark-gluon plasma will be reviewed in Chap. 7.

The linearly rising term in the potential makes it energetically impossible to separate a $q-\bar{q}$ pair. If the pair is created at one space-time point, for example in $e^{+} e^{-}$annihilation, and then the quark and the antiquark start moving away from each other in the center of mass frame, it soon becomes energetically favourable to create additional pairs, smoothly distributed in rapidity between the two leading charges, which neutralise colour and allow the final state to be reorganised into two jets of colourless hadrons, that communicate in the central region by a number of "wee" hadrons with small energy. It is just like the familiar example of the broken magnet: if you try to isolate a magnetic pole by stretching a dipole, the magnet breaks down and two new poles appear at the breaking point.

Confinement is essential to explain why nuclear forces have very short range while massless gluon exchange would be long range. Nucleons are colour singlets and they cannot exchange colour octet gluons but only colourless states. The lightest colour singlet hadronic particles are pions. So the range of nuclear forces is fixed by the pion mass $r \simeq m_{\pi}^{-1} \simeq 10^{-13} \mathrm{~cm}: V \approx \exp \left(-m_{\pi} r\right) / r$.

Why $S U\left(N_{C}=3\right)_{\text {colour }}$ ? The selection of $S U(3)$ as colour gauge group is unique in view of a number of constraints. (a) The group must admit complex representations because it must be able to distinguish a quark from an antiquark. In fact there are meson states made up of $q \bar{q}$ but not analogous $q q$ bound states. Among simple groups this restricts the choice to $S U(N)$ with $N \geq 3, S O(4 N+2)$ with $N \geq 2$ (taking into account that $S O(6)$ has the same algebra as $S U(4)$ ) and $E(6)$. (b) The group must admit a completely antisymmetric colour singlet baryon made up of 3 quarks: $q q q$. In fact, from the study of hadron spectroscopy we know that the low lying baryons, completing an octet and a decuplet of (flavour) $S U$ (3) (the approximate symmetry that rotate the three light quarks $\mathrm{u}, \mathrm{d}$ and s), are made up of three quarks and are colour singlets. The $q q q$ wave function must be completely antisymmetric in colour in order to agree with Fermi statistics. Indeed if we consider, for example, a $N^{*++}$ with spin z-component $+3 / 2$, this is made up of $(u \Uparrow u \Uparrow u \Uparrow)$ in an s-state. Thus its wave function is totally symmetric in space, spin and flavour so that complete antisymmetry in colour is required by Fermi 
statistics. In QCD this requirement is very simply satisfied by $\epsilon_{a b c} q^{a} q^{b} q^{c}$ where a, b, c are $S U(3)_{\text {colour }}$ indices. (c) The choice of $S U\left(N_{C}=3\right)_{\text {colour }}$ is confirmed by many processes that directly measure $N_{C}$. Some examples are listed here. The total rate for hadronic production in $e^{+} e^{-}$annihilation is linear in $N_{C}$. Precisely if we consider $R=\sigma\left(e^{+} e^{-} \rightarrow\right.$ hadrons $) / \sigma_{\text {point }}\left(e^{+} e^{-} \rightarrow \mu^{+} \mu^{-}\right)$above $b \bar{b}$ threshold and below $m_{Z}$ and we neglect small computable radiative corrections (that will be discussed later in Sect. 4.5) we have a sum of individual contributions (proportional to $Q^{2}$, where $Q$ is the electric charge in units of the proton charge) from $q \bar{q}$ final states with $q=u, c, d, s, b$ :

$$
R \approx N_{C}\left[2 \cdot \frac{4}{9}+3 \cdot \frac{1}{9}\right] \approx N_{C} \frac{11}{9}
$$

The data neatly indicate $N_{C}=3$ as seen from Fig. 4.2 [9]. The slight excess of the data with respect to the value $11 / 3$ is due to the QCD radiative corrections (Sect.4.5). Similarly we can consider the branching ratio $B\left(W^{-} \rightarrow e^{-} \bar{v}\right)$, again in Born approximation. The possible fermion-antifermion $(f \bar{f})$ final states are for $f=e^{-}, \mu^{-}, \tau^{-}, d, s$ (there is no $f=b$ because the top quark is too heavy for $b \bar{t}$ to occur). Each channel gives the same contribution, except that for quarks we have $N_{C}$ colours:

$$
B\left(W^{-} \rightarrow e^{-\bar{v}}\right) \approx \frac{1}{3+2 N_{C}}
$$

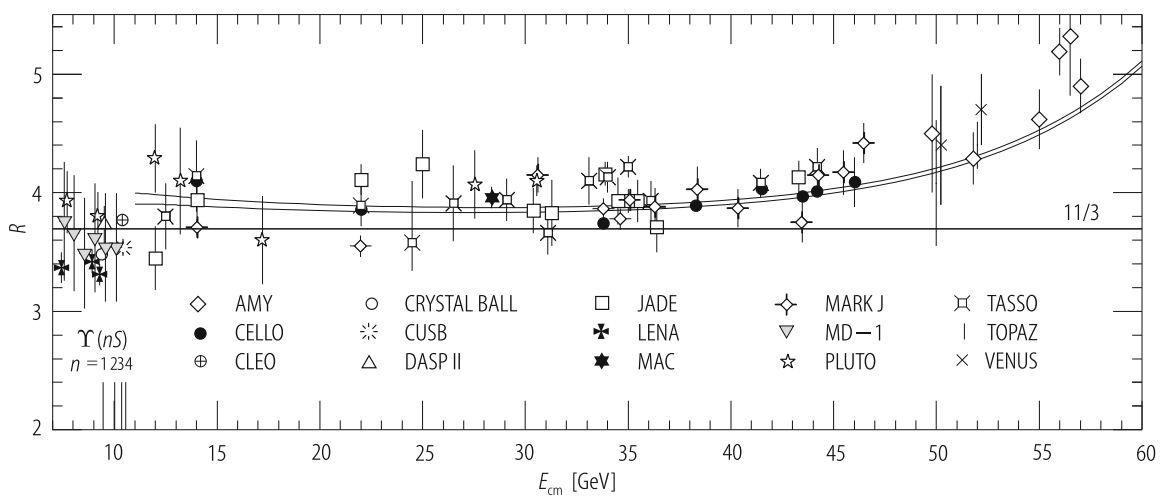

Fig. 4.2 Comparison of the data on $R=\sigma\left(e^{+} e^{-} \rightarrow\right.$ hadrons $) / \sigma_{\text {point }}\left(e^{+} e^{-} \rightarrow \mu^{+} \mu^{-}\right)$with the QCD prediction [9]. $N_{C}=3$ is indicated 
For $N_{C}=3$ we obtain $B=11 \%$ and the experimental number is $B=10.7 \%$. Another analogous example is the branching ratio $B\left(\tau^{-} \rightarrow e^{-} \overline{\nu_{e}} \nu_{\tau}\right)$. From the final state channels with $f=e^{-}, \mu^{-}, d$ we find

$$
B\left(\tau^{-} \rightarrow e^{-} \overline{\nu_{e}} \nu_{\tau}\right) \approx \frac{1}{2+N_{C}}
$$

For $N_{C}=3$ we obtain $B=20 \%$ and the experimental number is $B=18 \%$ (the less accuracy in this case is explained by the larger radiative and phase-space corrections because the mass of $\tau^{-}$is much smaller than $m_{W}$ ). An important process that is quadratic in $N_{C}$ is the rate $\Gamma\left(\pi^{0} \rightarrow 2 \gamma\right)$. This rate can be reliably calculated from a solid theorem in field theory which has to do with the chiral anomaly:

$$
\Gamma\left(\pi^{0} \rightarrow 2 \gamma\right) \approx\left(\frac{N_{C}}{3}\right)^{2} \frac{\alpha^{2} m_{\pi^{0}}^{3}}{32 \pi^{3} f_{\pi}^{2}}=(7.73 \pm 0.04)\left(\frac{N_{C}}{3}\right)^{2} \mathrm{eV}
$$

where the prediction is obtained for $f_{\pi}=(130.7 \pm 0.37) \mathrm{MeV}$. The experimental result is $\Gamma=(7.7 \pm 0.5) \mathrm{eV}$ in remarkable agreement with $N_{C}=3$. There are many more experimental confirmations that $N_{C}=3$ : for example the rate for Drell-Yan processes (see Sect. 5.4) is inversely proportional to $N_{C}$.

How do we get testable predictions from QCD? On the one hand there are non perturbative methods. The most important at present is the technique of lattice simulations: it is based on first principles, it has produced very valuable results on confinement, phase transitions, bound states, hadronic matrix elements and so on, and it is by now an established basic tool. The main limitation is from computing power and therefore there is continuous progress and a lot of good perspectives for the future. Another class of approaches is based on effective lagrangians which provide simpler approximations than the full theory, valid in some definite domain of physical conditions. Chiral lagrangians are based on soft pion theorems and are valid for suitable processes at energies below $1 \mathrm{GeV}$. Heavy quark effective theories are obtained from expanding in inverse powers of the heavy quark mass and are mainly important for the study of b and, to less accuracy, $c$ decays. The approach of QCD sum rules has led to interesting results but appears to offer not much potential for further development. Similarly specific potential models for quarkonium have a limited range of application. On the other hand, the perturbative approach, based on asymptotic freedom, still remains the main quantitative connection to experiment, due to its wide range of applicability to all sorts of "hard" processes. To perturbative QCD will be devoted the next sections.

\subsection{Massless QCD and Scale Invariance}

As discussed in Chap. 2, the QCD lagrangian in Eq. (4.1) only specifies the theory at the classical level. The procedure for quantisation of gauge theories involves a number of complications that arise from the fact that not all degrees of freedom of 
gauge fields are physical because of the constraints from gauge invariance which can be used to eliminate the dependent variables. This is already true for abelian theories and we are familiar with the QED case. One introduces a gauge fixing term (an additional term in the lagrangian density that acts as a Lagrange multiplier in the action extremisation). One can choose to preserve manifest Lorentz invariance. In this case, one adopts a covariant gauge, like the Lorentz gauge, and in QED one proceeds according to the formalism of Gupta-Bleuler. Or one can give up explicit formal covariance and work in a non covariant gauge, like the Coulomb or the axial gauges, and only quantise the physical degrees of freedom (in QED the transverse components of the photon field). While this is all for an abelian gauge theory, in the non-abelian case some additional complications arise, in particular the necessity to introduce ghosts for the formulation of Feynman rules. As we have seen, there are in general as many ghost fields as gauge bosons and they appear in the form of a transformation Jacobian in the Feynman diagram functional integral. Ghosts only propagate in closed loops and their vertices with gluons can be included as additional terms in the lagrangian density which are fixed once the gauge fixing terms and their infinitesimal gauge transformations are specified. Finally the complete Feynman rules in a given gauge can be obtained and they appear in Fig. 4.1.

Once the Feynman rules are derived we have a formal perturbative expansion but loop diagrams generate infinities. First a regularisation must be introduced, compatible with gauge symmetry and Lorentz invariance. This is possible in QCD. In principle one can introduce a cut-off $K$ (with dimensions of energy), for example, a' la Pauli-Villars. But at present the universally adopted regularisation procedure is dimensional regularisation that we will briefly describe later on. After regularisation the next step is renormalisation. In a renormalisable theory (like for all gauge theories in four spacetime dimensions and for QCD in particular) the dependence on the cutoff can be completely reabsorbed in a redefinition of particle masses, of gauge coupling(s) and of wave function normalisations. After renormalisation is achieved the perturbative definition of the quantum theory that corresponds to a classical lagrangian like in Eq. (4.1) is completed. In the QCD Lagrangian of Eq. (4.1) quark masses are the only parameters with physical dimensions (we work in the natural system of units $\hbar=c=1$ ). Naively we would expect that massless QCD is scale invariant. This is actually true at the classical level. Scale invariance implies that dimensionless observables should not depend on the absolute scale of energy but only on ratios of energy-dimensional variables. The massless limit should be relevant for the asymptotic large energy limit of processes which are non singular for $m \rightarrow 0$.

The naive expectation that massless QCD should be scale invariant is false in the quantum theory. The scale symmetry of the classical theory is unavoidably destroyed by the regularisation and renormalisation procedure which introduce a dimensional parameter in the quantum version of the theory. When a symmetry of the classical theory is necessarily destroyed by quantisation, regularisation and renormalisation one talks of an "anomaly". So, in this sense, scale invariance in massless QCD is anomalous. 
While massless QCD is finally not scale invariant, the departures from scaling are asymptotically small, logarithmic and computable. In massive QCD there are additional mass corrections suppressed by powers of $\mathrm{m} / \mathrm{E}$, where $\mathrm{E}$ is the energy scale (for non singular processes in the limit $m \rightarrow 0$ ). At the parton level ( $q$ and g) we can conceive to apply the asymptotics from massless QCD to processes and observables (we use the word "processes" for both) with the following properties ("hard processes"). (a) All relevant energy variables must be large:

$$
E_{i}=z_{i} Q, \quad Q>>m_{j} ; \quad z_{i}: \text { scaling variables o(1) }
$$

(b) There should be no infrared singularities (one talks of "infrared safe" processes).

(c) The processes concerned must be finite for $m \rightarrow 0$ (no mass singularities). To possibly satisfy these criteria processes must be as "inclusive" as possible: one should include all final states with massless gluon emission and add all mass degenerate final states (given that quarks are massless also $q-\bar{q}$ pairs can be massless if "collinear", that is moving together in the same direction at the common speed of light).

In perturbative QCD one computes inclusive rates for partons (the fields in the lagrangian, that is, in QCD, quarks and gluons) and takes them as equal to rates for hadrons. Partons and hadrons are considered as two equivalent sets of complete states. This is called "global duality" and it is rather safe in the rare instance of a totally inclusive final state. It is less so for distributions, like distributions in the invariant mass M ("local duality") where it can be reliable only if smeared over a sufficiently wide bin in M.

Let us discuss more in detail infrared and collinear safety. Consider, for example, a quark virtual line that ends up into a real quark plus a real gluon (Fig. 4.3).

For the propagator we have:

$$
\text { propagator }=\frac{1}{(p+k)^{2}-m^{2}}=\frac{1}{2(p \cdot k)}=\frac{1}{2 E_{k} E_{p}} \cdot \frac{1}{1-\beta_{p} \cos \theta}
$$

Since the gluon is massless, $E_{k}$ can vanish and this corresponds to an infrared singularity. Remember that we have to take the square of the amplitude and integrate over the final state phase space, or, in this case, all together, $d E_{k} / E_{k}$. Indeed we get $1 / E_{k}^{2}$ from the squared amplitude and $d^{3} k / E_{k} \sim E_{k} d E_{k}$ from the phase space.

Fig. 4.3 The splitting of a virtual quark into a quark and a gluon

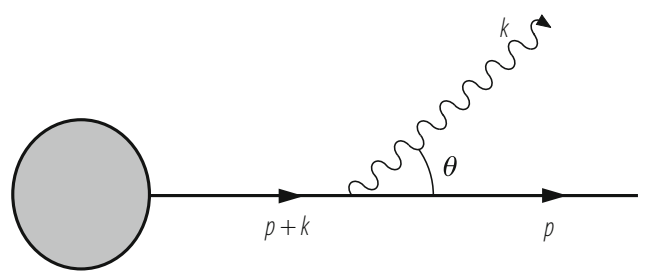




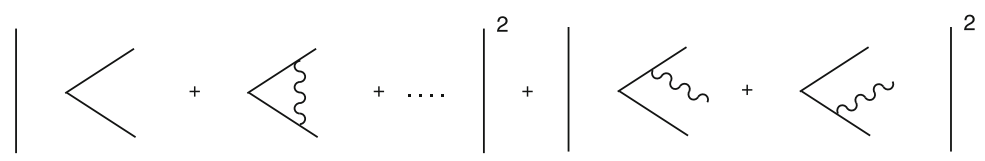

Fig. 4.4 The diagrams contributing to the total cross-section $e^{+} e^{-} \rightarrow$ hadrons at order $\alpha_{s}$. For simplicity, only the final state quarks and (virtual or real) gluons are drawn

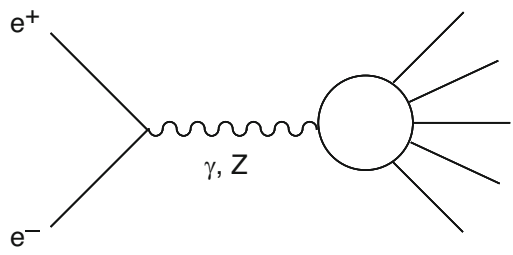

Fig. 4.5 The total cross-section $e^{+} e^{-} \rightarrow$ hadrons

Also, for $m \rightarrow 0, \beta_{p}=\sqrt{1-m^{2} / E_{p}^{2}} \rightarrow 1$ and $\left(1-\beta_{p} \cos \theta\right)$ vanishes at $\cos \theta=1$. This leads to a collinear mass singularity.

There are two very important theorems on infrared and mass singularities. The first one is the Bloch-Nordsieck theorem [8]: infrared singularities cancel between real and virtual diagrams (see Fig. 4.4) when all resolution indistinguishable final states are added up. For example, for each real detector there is a minimum energy of gluon radiation that can be detected. For the cancellation of infrared divergences, one should add all possible gluon emission with a total energy below the detectable minimum. The second one is the Kinoshita-Lee, Nauenberg theorem [10]: mass singularities connected with an external particle of mass $\mathrm{m}$ are canceled if all degenerate states (that is with the same mass) are summed up. That is for a final state particle of mass $\mathrm{m}$ we should add all final states that in the limit $m \rightarrow 0$ have the same mass, also including gluons and massless pairs. If a completely inclusive final state is taken, only the mass singularities from the initial state particles remain (we shall see that they will be absorbed inside the non perturbative parton densities, which are probability densities of finding the given parton in the initial hadron).

Hard processes to which the massless QCD asymptotics can possibly apply must be infrared and collinear safe, that is they must satisfy the requirements from the Bloch-Nordsieck and the Kinoshita-Lee-Nauenberg theorems. We give now some examples of important hard processes. One of the simplest hard processes is the totally inclusive cross section for hadron production in $e^{+} e^{-}$annihilation, Fig. 4.5, parameterised in terms of the already mentioned dimensionless observable $R=$ $\sigma\left(e^{+} e^{-} \rightarrow\right.$ hadrons $) / \sigma_{\text {point }}\left(e^{+} e^{-} \rightarrow \mu^{+} \mu^{-}\right)$. The pointlike cross section in the denominator is given by $\sigma_{\text {point }}=4 \pi \alpha^{2} / 3 s$, where $s=Q^{2}=4 E^{2}$ is the squared total center of mass energy and $Q$ is the mass of the exchanged virtual gauge boson. At parton level the final state is $\left(q \bar{q}+n g+n^{\prime} q^{\prime} \bar{q}^{\prime}\right)$ and $\mathrm{n}$ and $\mathrm{n}$ ' are limited at each order of perturbation theory. It is assumed that the conversion of partons into 
Fig. 4.6 Deep inelastic lepto-production

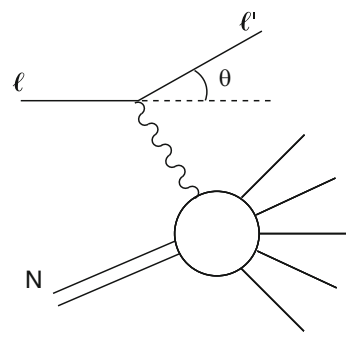

hadrons does not affect the rate (it happens with probability 1). We have already mentioned that in order for this to be true within a given accuracy an averaging over a sufficiently large bin of $Q$ must be understood. The binning width is larger in the vicinity of thresholds: for example when one goes across the charm $c \bar{c}$ threshold the physical cross-section shows resonance bumps which are absent in the smooth partonic counterpart which however gives an average of the cross-section.

A very important class of hard processes is Deep Inelastic Scattering (DIS)

$$
l+N \rightarrow l^{\prime}+X \quad l=e^{ \pm}, \mu^{ \pm}, \nu, \bar{v}
$$

which has played and still plays a very important role for our understanding of QCD and of nucleon structure. For the processes in Eq. (4.13), shown in Fig. 4.6, we have, in the lab system where the nucleon of mass $m$ is at rest:

$$
Q^{2}=-q^{2}=-\left(k-k^{\prime}\right)^{2}=4 E E^{\prime} \sin ^{2} \theta / 2 ; \quad m v=(p . q) ; \quad x=\frac{Q^{2}}{2 m v}
$$

In this case the virtual momentum $q$ of the gauge boson is spacelike. $x$ is the familiar Bjorken variable. The DIS processes in QCD will be extensively discussed in Sect. 4.5

\subsection{The Renormalisation Group and Asymptotic Freedom}

In this section we aim at providing a reasonably detailed introduction to the renormalisation group formalism and the concept of running coupling which leads to the result that QCD has the property of asymptotic freedom. We start with a summary on how renormalisation works.

In the simplest conceptual situation imagine that we implement regularisation of divergent integrals by introducing a dimensional cut-off $K$ that respects gauge and Lorentz invariance. The dependence of renormalised quantities on $K$ is eliminated by absorbing it into a redefinition of $\mathrm{m}$ (the quark mass: for simplicity we assume a single flavour here), the gauge coupling $e$ (can be $e$ in QED or $e_{s}$ in QCD) 
and the wave function renormalisation factors $Z_{q, g}^{1 / 2}$ for $\mathrm{q}$ and $\mathrm{g}$, using suitable renormalisation conditions (that is precise definitions of $\mathrm{m}, \mathrm{g}$ and $\mathrm{Z}$ that can be implemented order by order in perturbation theory). For example we can define the renormalised mass $\mathrm{m}$ as the position of the pole in the quark propagator and, similarly, the normalisation $Z_{q}$ as the residue at the pole:

$$
\text { Propagator }=\frac{Z_{q}}{p^{2}-m^{2}}+\text { no }- \text { pole terms }
$$

The renormalised coupling $e$ can be defined in terms of a renormalised 3-point vertex at some specified values of the external momenta. Precisely, we consider a one particle irreducible vertex (1PI). We recall that a connected Green function is the sum of all connected diagrams, while 1PI Green functions are the sum of all diagrams that cannot be separated into two disconnected parts by cutting only one line.

We now become more specific by concentrating on the case of massless QCD. If we start from a vanishing mass at the classical (or "bare") level, $m_{0}=0$, the mass is not renormalised because it is protected by a symmetry, chiral symmetry. The conserved currents of chiral symmetry are axial currents: $\bar{q} \gamma_{\mu} \gamma_{5} q$. The divergence of the axial current gives, by using the Dirac equation, $\partial^{\mu}\left(\bar{q} \gamma_{\mu} \gamma_{5} q\right)=2 m \bar{q} \gamma_{5} q$. So the axial current and the corresponding axial charge are conserved in the massless limit. Since QCD is a vector theory we have not to worry about chiral anomalies in this respect. So one can choose a regularisation that preserves chiral symmetry besides gauge and Lorentz symmetry. Then the renormalised mass remains zero. The renormalised propagator has the form in Eq. (4.15) with $m=0$.

The renormalised coupling $e_{s}$ can be defined from the renormalised 1PI 3-gluon vertex at a scale $-\mu^{2}$ (Fig. 4.7):

$$
V_{\text {bare }}\left(p^{2}, q^{2}, r^{2}\right)=Z V_{\text {ren }}\left(p^{2}, q^{2}, r^{2}\right), \quad Z=Z_{g}^{-3 / 2}, \quad V_{\text {ren }}\left(-\mu^{2},-\mu^{2},-\mu^{2}\right) \rightarrow e_{s}
$$

We could as well use the quark-gluon vertex or any other vertex which coincides with $e_{0}$ in lowest order (even the ghost-gluon vertex, if we want). With a regularisation and renormalisation that preserves gauge invariance we are guaranteed that all these different definitions are equivalent.

Here $V_{\text {bare }}$ is what is obtained from computing the Feynman diagrams including, for example, the 1-loop corrections at the lowest non trivial order ( $V_{\text {bare }}$ is defined

Fig. 4.7 Diagrams contributing to the $1 \mathrm{PI}$ 3-gluon vertex at the one-loop approximation level

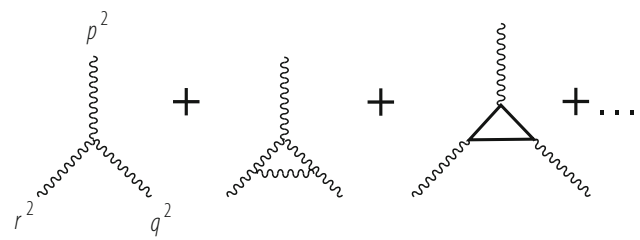


as the scalar function multiplying the vertex tensor, normalised in such a way that it coincides with $e_{s 0}$ in lowest order). $V_{\text {bare }}$ contains the cut-off $K$ but does not know about $\mu . Z$ is a factor that depends both on the cut-off and on $\mu$ but not on momenta. Because of infrared singularities the defining scale $\mu$ cannot vanish. The negative value $-\mu^{2}<0$ is chosen to stay away from physical cuts (a gluon with negative virtual mass cannot decay). Similarly, in the massless theory, we can define $Z_{g}^{-1}$ as the inverse gluon propagator (the 1PI 2-point function) at the same scale $-\mu^{2}$ (the vanishing mass of the gluon is guaranteed by gauge invariance).

After computing all 1-loop diagrams indicated in Fig. 4.7 we have:

$$
\begin{aligned}
V_{\text {bare }}\left(p^{2}, p^{2}, p^{2}\right) & =e_{0 s}\left[1+c \alpha_{0 s} \cdot \log \frac{K^{2}}{p^{2}}+\ldots\right]= \\
& =\left[1+c \alpha_{s} \cdot \log \frac{K^{2}}{-\mu^{2}}+\ldots\right] e_{0 s}\left[1+c \alpha_{s} \cdot \log \frac{-\mu^{2}}{p^{2}}+\ldots\right] \\
& =Z_{V}^{-1} e_{0 s}\left[1+c \alpha_{s} \cdot \log \frac{-\mu^{2}}{p^{2}}+\ldots\right] \\
& =\left[1+d \alpha_{s} \cdot \log \frac{K^{2}}{-\mu^{2}}+\ldots\right] e_{s}\left[1+c \alpha_{s} \cdot \log \frac{-\mu^{2}}{p^{2}}+\ldots\right] \\
& =Z_{g}^{-3 / 2} V_{\text {ren }}
\end{aligned}
$$

Note the replacement of $e_{0}$ with $e$ in the second step, compensated by changing $c$ into $d$ in the first bracket (corresponding to $e_{0}=Z_{g}^{-3 / 2} Z_{V} e$ ). The definition of $e_{s}$ demands that one precisely specifies what is included in $Z$. For this, in a given renormalisation scheme, a prescription is fixed to specify the finite terms that go into $\mathrm{Z}$ (i.e. the terms of order $\alpha_{s}$ that accompany $\log K^{2}$ ). Then $V_{\text {ren }}$ is specified and the renormalised coupling is defined from it according to Eq. (4.16). For example, in the momentum subtraction scheme we define $V_{\text {ren }}\left(p^{2}, p^{2}, p^{2}\right)=$ $e_{s}+V_{\text {bare }}\left(p^{2}, p^{2}, p^{2}\right)-V_{\text {bare }}\left(-\mu^{2},-\mu^{2},-\mu^{2}\right)$, which is equivalent to say, at 1-loop, that all finite terms that do not vanish at $p^{2}=-\mu^{2}$ are included in $\mathrm{Z}$.

A crucial observation is that $V_{\text {bare }}$ depends on $K$ but not on $\mu$, which is only introduced when $\mathrm{Z}, V_{\text {ren }}$ and hence $\alpha_{s}$ are defined. (From here on, for shorthand, we write $\alpha$ to indicate either the QED coupling or the QCD coupling $\alpha_{s}$ ). More in general for a generic Green function $\mathrm{G}$, we similarly have:

$$
G_{\text {bare }}\left(K^{2}, \alpha_{0}, p_{i}^{2}\right)=Z_{G} G_{r e n}\left(\mu^{2}, \alpha, p_{i}^{2}\right)
$$

so that we have:

$$
\frac{d G_{\text {bare }}}{d \log \mu^{2}}=\frac{d}{d \log \mu^{2}}\left[Z_{G} G_{r e n}\right]=0
$$


or

$$
Z_{G}\left[\frac{\partial}{\partial \log \mu^{2}}+\frac{\partial \alpha}{\partial \log \mu^{2}} \frac{\partial}{\partial \alpha}+\frac{1}{Z_{G}} \frac{\partial Z_{G}}{\partial \log \mu^{2}}\right] G_{r e n}=0
$$

Finally the renormalisation group equation (RGE) can be written as:

$$
\left[\frac{\partial}{\partial \log \mu^{2}}+\beta(\alpha) \frac{\partial}{\partial \alpha}+\gamma_{G}(\alpha)\right] G_{r e n}=0
$$

where

$$
\beta(\alpha)=\frac{\partial \alpha}{\partial \log \mu^{2}}
$$

and

$$
\gamma_{G}(\alpha)=\frac{\partial \log Z_{G}}{\partial \log \mu^{2}}
$$

Note that $\beta(\alpha)$ does not depend on which Green function $G$ we are considering, but it is a property of the theory and the renormalisation scheme adopted, while $\gamma_{G}(\alpha)$ also depends on $G$. Strictly speaking the RGE as written above is only valid in the Landau gauge $(\lambda=0)$. In other gauges an additional term that takes the variation of the gauge fixing parameter $\lambda$ should also be included. We omit this term, for simplicity, as it is not relevant at the 1-loop level.

Assume that we want to apply the RGE to some hard process at a large scale $\mathrm{Q}$, related to a Green function $\mathrm{G}$ that we can always take as dimensionless (by multiplication by a suitable power of Q). Since the interesting dependence on $\mathrm{Q}$ will be logarithmic we introduce the variable $t$ as :

$$
t=\log \frac{Q^{2}}{\mu^{2}}
$$

Then we can write $G_{r e n} \equiv F\left(t, \alpha, x_{i}\right)$ where $x_{i}$ are scaling variables (we often omit to write them in the following). In the naive scaling limit $F$ should be independent of $t$. To find the actual dependence on $t$, we want to solve the RGE

$$
\left[-\frac{\partial}{\partial t}+\beta(\alpha) \frac{\partial}{\partial \alpha}+\gamma_{G}(\alpha)\right] G_{r e n}=0
$$

with a given boundary condition at $t=0\left(\right.$ or $\left.Q^{2}=\mu^{2}\right)$ : $F(0, \alpha)$.

We first solve the RGE in the simplest case that $\gamma_{G}(\alpha)=0$. This is not an unphysical case: for example, it applies to $R_{e^{+}} e^{-}$where the vanishing of $\gamma$ is related to the non renormalisation of the electric charge in QCD (otherwise the proton and 
the electron charge would not exactly compensate: this will be better explained in Sect. 4.5). So we consider the equation:

$$
\left[-\frac{\partial}{\partial t}+\beta(\alpha) \frac{\partial}{\partial \alpha}\right] G_{r e n}=0
$$

The solution is simply

$$
F(t, \alpha)=F[0, \alpha(t)]
$$

where the "running coupling" $\alpha(t)$ is defined by:

$$
t=\int_{\alpha}^{\alpha(t)} \frac{1}{\beta\left(\alpha^{\prime}\right)} d \alpha^{\prime}
$$

Note that from this definition it follows that $\alpha(0)=\alpha$, so that the boundary condition is also satisfied. To prove that $F[0, \alpha(t)]$ is indeed the solution, we first take derivatives with respect of $\mathrm{t}$ and $\alpha$ (the two independent variables) of both sides of Eq. (4.28). By taking $d / d t$ we obtain

$$
1=\frac{1}{\beta(\alpha(t)} \frac{\partial \alpha(t)}{\partial t}
$$

We then take $d / d \alpha$ and obtain

$$
0=-\frac{1}{\beta(\alpha)}+\frac{1}{\beta(\alpha(t)} \frac{\partial \alpha(t)}{\partial \alpha}
$$

These two relations make explicit the dependence of the running coupling on $\mathrm{t}$ and $\alpha$ :

$$
\begin{aligned}
& \frac{\partial \alpha(t)}{\partial t}=\beta(\alpha(t)) \\
& \frac{\partial \alpha(t)}{\partial \alpha}=\frac{\beta(\alpha(t))}{\beta(\alpha)}
\end{aligned}
$$

Using these two equations one immediately checks that $F[0, \alpha(t)]$ is indeed the solution.

Similarly, one finds that the solution of the more general equation with $\gamma \neq 0$, Eq. (4.25), is given by:

$$
F(t, \alpha)=F[0, \alpha(t)] \exp \int_{\alpha}^{\alpha(t)} \frac{\gamma\left(\alpha^{\prime}\right)}{\beta\left(\alpha^{\prime}\right)} d \alpha^{\prime}
$$


In fact the sum of the two derivatives acting on the factor $F[0, \alpha(t)]$ vanishes and the exponential is by itself a solution of the complete equation. Note that the boundary condition is also satisfied.

The important point is the appearance of the running coupling that determines the asymptotic departures from scaling. The next step is to study the functional form of the running coupling. From Eq. (4.31) we see that the rate of change with the running coupling is determined by the $\beta$ function. In turn $\beta(\alpha)$ is determined by the $\mu$ dependence of the renormalised coupling through Eq. (4.22). Clearly there is no dependence on $\mu$ of the basic 3 -gluon vertex in lowest order (order $e$ ). The dependence starts at 1-loop, that is at order $e^{3}$ (one extra gluon has to be emitted and reabsorbed). Thus we obtain that in perturbation theory:

$$
\frac{\partial e}{\partial \log \mu^{2}} \propto e^{3}
$$

Recalling that $\alpha=e^{2} / 4 \pi$, we have:

$$
\frac{\partial \alpha}{\partial \log \mu^{2}} \propto 2 e \frac{\partial e}{\partial \log \mu^{2}} \propto e^{4} \propto \alpha^{2}
$$

Thus the behaviour of $\beta(\alpha)$ in perturbation theory is as follows:

$$
\beta(\alpha)= \pm b \alpha^{2}\left[1+b^{\prime} \alpha+\ldots\right]
$$

Since the sign of the leading term is crucial in the following discussion, we stipulate that always $b>0$ and we make the sign explicit in front.

Let us make the procedure for computing the 1-loop beta function in QCD (or, similarly, in QED) more precise. The result of the 1loop 1PI diagrams for $V_{\text {ren }}$ can be written down as (we denote $e_{s}$ and $\alpha_{s}$ by $e$ and $\alpha$, for shorthand):

$$
V_{\text {ren }}=e\left[1+\alpha B_{3 g} \log \frac{\mu^{2}}{-p^{2}}+\ldots\right]
$$

$V_{\text {ren }}$ satisfies the RGE:

$$
\left[\frac{\partial}{\partial \log \mu^{2}}+\beta(\alpha) \frac{\partial e}{\partial \alpha} \frac{\partial}{\partial e}-\frac{3}{2} \gamma_{g}(\alpha)\right] V_{r e n}=0
$$

With respect to Eq. (4.21) the beta function term has been rewritten taking into account that $V_{\text {ren }}$ starts with $e$ and the anomalous dimension term arises from a factor $Z_{g}^{-1 / 2}$ for each gluon leg. In general for a n-leg 1PI Green function $V_{n, \text { bare }}=Z_{g}^{-n / 2} V_{n, \text { ren }}$, if all external legs are gluons. Note that in the particular case of $V=V_{3}$ that is used to define $e$ other $\mathrm{Z}$ factors are absorbed in the replacement 
$Z_{V}^{-1} Z_{g}^{3 / 2} e_{0}=e$. At 1-loop accuracy we replace $\beta(\alpha)=-b \alpha^{2}$ and $\gamma_{g}(\alpha)=\gamma_{g}^{(1)} \alpha$. All together one obtains:

$$
b=2\left(B_{3 g}-\frac{3}{2} \gamma_{g}^{(1)}\right)
$$

Similarly we can write the diagrammatic expression and the RGE for the 1PI 2gluon Green function which is the inverse gluon propagator $\Pi$ (a scalar function after removing the gauge invariant tensor):

$$
\Pi_{r e n}=\left[1+\alpha B_{2 g} \log \frac{\mu^{2}}{-p^{2}}+\ldots\right]
$$

and

$$
\left[\frac{\partial}{\partial \log \mu^{2}}+\beta(\alpha) \frac{\partial}{\partial \alpha}-\gamma_{g}(\alpha)\right] \Pi_{r e n}=0
$$

Notice that the normalisation and the phase of $\Pi$ are specified by the lowest order term being one. In this case the $\beta$ function term is negligible being of order $\alpha^{2}$ (because $\Pi$ is a function of $e$ only through $\alpha$ ). and we obtain:

$$
\gamma_{g}^{(1)}=B_{2 g}
$$

Thus, finally:

$$
b=2\left(B_{3 g}-\frac{3}{2} B_{2 g}\right)
$$

By direct calculation at 1-loop one finds:

$$
\text { QED : } \quad \beta(\alpha) \sim+b \alpha^{2}+\ldots . . \quad b=\sum_{i} \frac{N_{C} Q_{i}^{2}}{3 \pi}
$$

where $N_{C}=3$ for quarks and $N_{C}=1$ for leptons and the sum runs over all fermions of charge $Q_{i} e$ that are coupled. Also, one finds:

$$
\mathrm{QCD}: \quad \beta(\alpha) \sim-b \alpha^{2}+\ldots . . \quad b=\frac{11 N_{C}-2 n_{f}}{12 \pi}
$$

where, as usual, $n_{f}$ is the number of coupled flavours of quarks (we assume here that $n_{f} \leq 16$ so that $b>0$ in QCD). If $\alpha(t)$ is small we can compute $\beta(\alpha(t))$ in perturbation theory. The sign in front of $b$ then decides the slope of the coupling: $\alpha(t)$ increases with t (or $Q^{2}$ ) if $\beta$ is positive at small $\alpha$ (QED), or $\alpha(t)$ decreases with $\mathrm{t}$ (or $Q^{2}$ ) if $\beta$ is negative at small $\alpha$ (QCD). A theory like QCD where the running 
coupling vanishes asymptotically at large $Q^{2}$ is called (ultraviolet) "asymptotically free". An important result that has been proven is that in four spacetime dimensions all and only non-abelian gauge theories are asymptotically free.

Going back to Eq. (4.28) we replace $\beta(\alpha) \sim \pm b \alpha^{2}$, do the integral and perform a simple algebra. We find

$$
\text { QED : } \quad \alpha(t) \sim \frac{\alpha}{1-b \alpha t}
$$

and

$$
\mathrm{QCD}: \quad \alpha(t) \sim \frac{\alpha}{1+b \alpha t}
$$

A slightly different form is often used in QCD. Defining $1 / \alpha=b \log \mu^{2} / \Lambda_{Q C D}^{2}$ we can write:

$$
\alpha(t) \sim \frac{1}{\frac{1}{\alpha}+b t}=\frac{1}{b \log \frac{\mu^{2}}{\Lambda_{Q C D}^{2}}+b \log \frac{Q^{2}}{\mu^{2}}}=\frac{1}{b \log \frac{Q^{2}}{\Lambda_{Q C D}^{2}}}
$$

We see that $\alpha(t)$ decreases logarithmically with $Q^{2}$ and that one can introduce a dimensional parameter $\Lambda_{Q C D}$ that replaces $\mu$. Often in the following we will simply write $\Lambda$ for $\Lambda_{Q C D}$. Note that it is clear that $\Lambda$ depends on the particular definition of $\alpha$, not only on the defining scale $\mu$ but also on the renormalisation scheme (see, for example, the discussion in the next session). Through the parameter $b$, and in general through the $\beta$ function, it also depends on the number $n_{f}$ of coupled flavours. It is very important to note that QED and QCD are theories with "decoupling": up to the scale $Q$ only quarks with masses $m<<Q$ contribute to the running of $\alpha$. This is clearly very important, given that all applications of perturbative QCD so far apply to energies below the top quark mass $m_{t}$. For the validity of the decoupling theorem [11] it is necessary that the theory where all the heavy particle internal lines are eliminated is still renormalisable and that the coupling constants do not vary with the mass. These requirements are true for the mass of heavy quarks in QED and QCD, but are not true in the electroweak theory where the elimination of the top would violate $S U(2)$ symmetry (because the $t$ and $b$ left quarks are in a doublet) and the quark couplings to the Higgs multiplet (hence to the longitudinal gauge bosons) are proportional to the mass. In conclusion, in QED and QCD, quarks with $m>>Q$ do not contribute to $n_{f}$ in the coefficients of the relevant $\beta$ function. The effects of heavy quarks are power suppressed and can be taken separately into account. For example, in $e^{+} e^{-}$annihilation for $2 m_{c}<Q<2 m_{b}$ the relevant asymptotics is for $n_{f}=4$, while for $2 m_{b}<Q<2 m_{t} n_{f}=5$. Going accross the $b$ threshold the $\beta$ function coefficients change, so the $\alpha(t)$ slope changes. But $\alpha(t)$ is continuous, so that $\Lambda$ changes so as to keep constant $\alpha(t)$ at the matching point at $Q \sim o\left(2 m_{b}\right)$. The effect on $\Lambda$ is large: approximately $\Lambda_{5} \sim 0.65 \Lambda_{4}$. 
Note the presence of a pole in Eqs. $(4.46,4.47)$ at $\pm b \alpha t=1$, called the Landau pole, who realised its existence in QED already in the '50's. For $\mu \sim m_{e}$ (in QED) the pole occurs beyond the Planck mass. In QCD the Landau pole is located for negative $t$ or at $Q<\mu$ in the region of light hadron masses. Clearly the issue of the definition and the behaviour of the physical coupling (which is always finite, when defined in terms of some physical process) in the region around the perturbative Landau pole is a problem that lies outside the domain of perturbative QCD.

The non leading terms in the asymptotic behaviour of the running coupling can in principle be evaluated going back to Eq. (4.36) and computing $b^{\prime}$ at 2-loops and so on. But in general the perturbative coefficients of $\beta(\alpha)$ depend on the definition of the renormalised coupling $\alpha$ (the renormalisation scheme), so one wonders whether it is worthwhile to do a complicated calculation to get $b^{\prime}$ if then it must be repeated for a different definition or scheme. In this respect it is interesting to remark that actually both $b$ and $b^{\prime}$ are independent of the definition of $\alpha$, while higher order coefficients do depend on that. Here is the simple proof. Two different perturbative definitions of $\alpha$ are related by $\alpha^{\prime} \sim \alpha\left(1+c_{1} \alpha+\ldots\right)$. Then we have:

$$
\begin{aligned}
\beta\left(\alpha^{\prime}\right)=\frac{d \alpha^{\prime}}{d \log \mu^{2}} & =\frac{d \alpha}{d \log \mu^{2}}\left(1+2 c_{1} \alpha+\ldots\right) \\
& = \pm b \alpha^{2}\left(1+b^{\prime} \alpha+\ldots\right)\left(1+2 c_{1} \alpha+\ldots\right) \\
& = \pm b \alpha^{\prime 2}\left(1+b^{\prime} \alpha^{\prime}+\ldots\right)
\end{aligned}
$$

which shows that, up to the first subleading order, $\beta\left(\alpha^{\prime}\right)$ has the same form as $\beta(\alpha)$.

In QCD $\left(N_{C}=3\right)$ one has calculated:

$$
b^{\prime}=\frac{153-19 n_{f}}{2 \pi\left(33-2 n_{f}\right)}
$$

By taking $b^{\prime}$ into account one can write the expression of the running coupling at next to the leading order (NLO):

$$
\alpha\left(Q^{2}\right)=\alpha_{L O}\left(Q^{2}\right)\left[1-b^{\prime} \alpha_{L O}\left(Q^{2}\right) \log \log \frac{Q^{2}}{\Lambda^{2}}+\ldots\right]
$$

where $\alpha_{L O}^{-1}=b \log Q^{2} / \Lambda^{2}$ is the LO result (actually at NLO the definition of $\Lambda$ is modified according to $b \log \mu^{2} / \Lambda^{2}=1 / \alpha+b^{\prime} \log b \alpha$ ).

Summarizing, we started from massless classical QCD which is scale invariant. But we have seen that the procedure of quantisation, regularisation and renormalisation necessarily breaks scale invariance. In the quantum QCD theory there is a scale of energy, $\Lambda$, which from experiment is of the order of a few hundred $\mathrm{MeV}$, its precise value depending on the definition, as we shall see in detail. Dimensionless quantities depend on the energy scale through the running coupling which is a logarithmic function of $Q^{2} / \Lambda^{2}$. In QCD the running coupling decreases 
logarithmically at large $Q^{2}$ (asymptotic freedom), while in QED the coupling has the opposite behaviour.

\subsection{More on the Running Coupling}

In the previous section we have introduced the renormalised coupling $\alpha$ in terms of the 3-gluon vertex at $p^{2}=-\mu^{2}$ (momentum subtraction). The Ward identities of QCD then ensure that the coupling defined from other vertices like the $\bar{q} q g$ vertex are renormalised in the same way and the finite radiative corrections are related. But at present the universally adopted definition of $\alpha_{s}$ is in terms of dimensional regularisation because of computational simplicity which is essential given the great complexity of present day calculations. So we now briefly review the principles of dimensional regularisation and the definition of Minimal Subtraction $(M S)$ and Modified Minimal Subtraction $(\overline{M S})$. The $\overline{M S}$ definition of $\alpha_{s}$ is the one most commonly adopted in the literature and a value quoted for it is nomally referring to this definition.

Dimensional Regularisation (DR) is a gauge and Lorentz invariant regularisation that consists in formulating the theory in $D<4$ spacetime dimensions in order to make loop integrals ultraviolet finite. In DR one rewrites the theory in D dimensions ( $\mathrm{D}$ is integer at the beginning, but then we will see that the expression of diagrams makes sense at all D except for isolated singularities). The metric tensor is extended into a $D \times D$ matrix $g_{\mu \nu}=\operatorname{diag}(1,-1,-1, \ldots,-1)$ and 4-vectors are given by $k^{\mu}=\left(k^{0}, k^{1}, \ldots, k^{D-1}\right)$. The Dirac $\gamma^{\mu}$ are $f(D) \times f(D)$ matrices and it is not important what is the precise form of the function $f(D)$. It is sufficient to extend the usual algebra in a straightforward way like $\left\{\gamma_{\mu}, \gamma_{\nu}\right\}=2 g_{\mu, v} I$, with $I$ the Ddimensional identity matrix, $\gamma^{\mu} \gamma^{\nu} \gamma_{\mu}=-(D-2) \gamma^{\nu}$ or $\operatorname{Tr}\left(\gamma^{\mu} \gamma^{\nu}\right)=f(D) g_{\mu \nu}$.

The physical dimensions of fields change in $\mathrm{D}$ dimensions and, as a consequence, the gauge couplings become dimensional $e_{D}=\mu^{\epsilon} e$, where $e$ is dimensionless, $D=4-2 \epsilon$ and $\mu$ is a scale of mass (this is how a scale of mass is introduced in the DR of massless QCD!). The dimension of fields is determined by requiring that the action $S=\int d^{D} x \mathcal{L}$ is dimensionless. By inserting for $\mathcal{L}$ terms like $m \bar{\Psi} \Psi$ or $m^{2} \phi^{\dagger} \phi$ or $e \bar{\Psi} \gamma^{\mu} \Psi A_{\mu}$ the dimensions of the fields and coupling are determined as: $m, \Psi, \phi, A_{\mu}, e=1,(D-1) / 2,(D-2) / 2,(D-2) / 2,(4-D) / 2$, respectively. The formal expression of loop integrals can be written for any D. For example:

$$
\int \frac{d^{D} k}{(2 \pi)^{D}} \frac{1}{\left(k^{2}-m^{2}\right)^{2}}=\frac{\Gamma(2-D / 2)\left(-m^{2}\right)^{D / 2-2}}{(4 \pi)^{D / 2}}
$$

For $D=4-2 \epsilon$ one can expand using:

$$
\Gamma(\epsilon)=\frac{1}{\epsilon}-\gamma_{E}+o(\epsilon), \quad \gamma_{E}=0.5772 \ldots .
$$


For some Green function $\mathrm{G}$, normalised to one in lowest order, (like V/e with $\mathrm{V}$ the 3 -g vertex function at the symmetric point $p^{2}=q^{2}=r^{2}$, considered in the previous section) we typically find at 1-loop:

$$
G_{\text {bare }}=1+\alpha_{0}\left(\frac{-\mu^{2}}{p^{2}}\right)^{\epsilon}\left[B\left(\frac{1}{\epsilon}+\log 4 \pi-\gamma_{E}\right)+A+o(\epsilon)\right]
$$

In $\overline{M S}$ one rewrites this at 1-loop accuracy (diagram by diagram: this is a virtue of the method):

$$
\begin{aligned}
G_{\text {bare }} & =Z G_{\text {ren }} \\
Z & =1+\alpha\left[B\left(\frac{1}{\epsilon}+\log 4 \pi-\gamma_{E}\right)\right] \\
G_{r e n} & =1+\alpha\left[B \log \frac{-\mu^{2}}{p^{2}}+A\right]
\end{aligned}
$$

Here $Z$ stands for the relevant product of renormalisation factors. In the original $M S$ prescription only $1 / \epsilon$ was subtracted (that clearly plays the role of a cutoff) and not also $\log 4 \pi$ and $\gamma_{E}$. Later, since these constants always appear from the expansion of $\Gamma$ functions it was decided to modify $M S$ into $\overline{M S}$. Note that the $\overline{M S}$ definition of $\alpha$ is different than that in the momentum subtraction scheme because the finite terms (those beyond logs) are different. In particular here $\delta G_{r e n}$ does not vanish at $p^{2}=-\mu^{2}$.

The third [12] and fourth [13] coefficients of the QCD $\beta$ function are also known in the $\overline{M S}$ prescription (recall that only the first two coefficients are scheme independent). The calculation of the last term involved the evaluation of some 50,000 4-loop diagrams. Translated in numbers, for $n_{f}=5$ one obtains :

$$
\beta(\alpha)=-0.610 \alpha^{2}\left[1+1.261 \ldots \frac{\alpha}{\pi}+1.475 \ldots\left(\frac{\alpha}{\pi}\right)^{2}+9.836 \ldots\left(\frac{\alpha}{\pi}\right)^{3} \ldots\right]
$$

It is interesting to remark that the expansion coefficients are all of order 1 or (10 for the last one), so that the $\overline{M S}$ expansion looks reasonably well behaved.

It is important to keep in mind that the QED and QCD perturbative series, after renormalisation, have all their coefficients finite, but the expansion does not converge. Actually the perturbative series are not even Borel summable. After Borel resummation for a given process one is left with a result which is ambiguous by terms typically down by $\exp -n /(b \alpha)$, with $\mathrm{n}$ an integer and $\mathrm{b}$ the first $\beta$ function coefficient. In QED these corrective terms are extremely small and not very important in practice. On the contrary in QCD $\alpha=\alpha_{S}\left(Q^{2}\right) \sim 1 /\left(b \log Q^{2} / \Lambda^{2}\right)$ and the ambiguous terms are of order $\left(1 / Q^{2}\right)^{n}$, that is are power suppressed. It is interesting that, through this mechanism, the perturbative version of the theory is able to somehow take into account the power suppressed corrections. A sequence 
of diagrams with factorial growth at large order $\mathrm{n}$ is made up by dressing gluon propagators by any number of quark bubbles together with their gauge completions (renormalons).The problem of the precise relation between the ambiguities of the perturbative expansion and the higher twist corrections has been discussed in recent years [14].

\subsection{Application to Hard Processes}

\subsection{1 $R_{e^{+} e^{-}}$and Related Processes}

The simplest hard process is $R_{e^{+}} e^{-}$that we have already started to discuss. $R$ is dimensionless and in perturbation theory is given by $R=N_{C} \sum_{i} Q_{i}^{2} F\left(t, \alpha_{s}\right)$, where $F=1+o\left(\alpha_{S}\right)$. We have already mentioned that for this process the "anomalous dimension" function vanishes: $\gamma\left(\alpha_{s}\right)=0$ because of electric charge non renormalisation by strong interactions. Let us review how this happens in detail. The diagrams that are relevant for charge renormalisation in QED at 1-loop are shown in Fig. 4.8. The Ward identity that follows from gauge invariance in QED imposes that the vertex $\left(Z_{V}\right)$ and the self-energy $\left(Z_{f}\right)$ renormalisation factors cancel and the only divergence remains in $Z_{\gamma}$, the vacuum polarization of the photon. So the charge is only renormalised by the photon blob, hence it is universal (the same factor for all fermions, independent of their charge) and is not affected by QCD at 1-loop. It is true that at higher orders the photon vacuum polarization diagram is affected by QCD (for example, at 2-loops we can exchange a gluon between the quarks in the photon loop) but the renormalisation induced by the vacuum polarisation diagram remains independent of the nature of the fermion to which the photon line is attached. The gluon contributions to the vertex $\left(Z_{V}\right)$ and to the

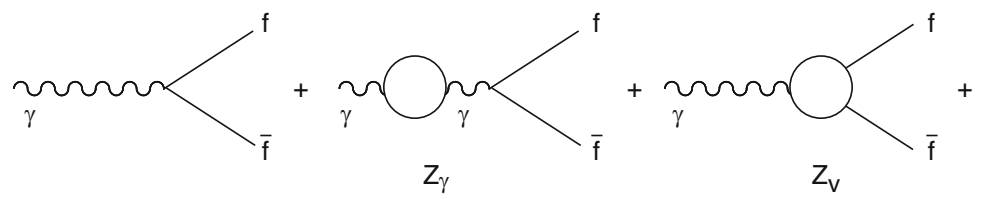

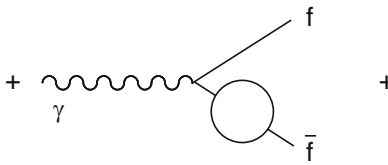

$Z_{f}$

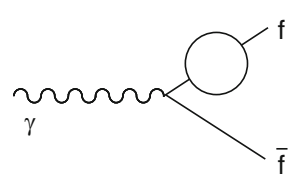

$Z_{f}$

Fig. 4.8 Diagrams for charge renormalisation in QED at 1-loop 

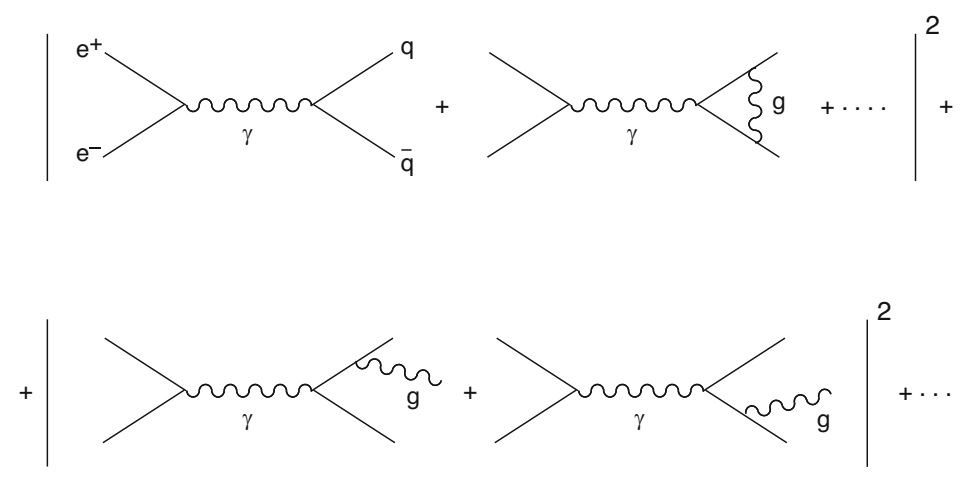

Fig. 4.9 Real and virtual diagrams relevant for the computation of $\mathrm{R}$ at 1-loop accuracy

self-energy $\left(Z_{f}\right)$ cancel because they have exactly the same structure as in QED, so that $\gamma\left(\alpha_{s}\right)=0$.

At 1-loop the diagrams relevant for the computation of $\mathrm{R}$ are shown in Fig. 4.9. There are virtual diagrams and real diagrams with one additional gluon in the final state. Infrared divergences cancel between the interference term of the virtual diagrams and the absolute square of the real diagrams, according to the BlochNordsieck theorem. Similarly there are no mass singularities, in agreement with the Kinoshita-Lee-Nauenberg theorem, because the initial state is purely leptonic and all degenerate states that can appear at the given order are included in the final state. Given that $\gamma\left(\alpha_{s}\right)=0$ the RGE prediction is simply given, as we have already seen, by $F\left(t, \alpha_{s}\right)=F\left[0, \alpha_{s}(t)\right]$. This means that if we do, for example, a 2-loop calculation, we must obtain a result of the form:

$$
F\left(t, \alpha_{s}\right)=1+c_{1} \alpha_{s}\left(1-b \alpha_{s} t\right)+c_{2} \alpha_{s}^{2}+o\left(\alpha_{s}^{3}\right)
$$

In fact we see that this form, taking into account that from Eq. (4.47) we have:

$$
\alpha_{s}(t) \sim \frac{\alpha_{s}}{1+b \alpha_{s} t} \sim \alpha_{s}\left(1-b \alpha_{s} t+\ldots\right)
$$

can be rewritten as

$$
F\left(t, \alpha_{s}\right)=1+c_{1} \alpha_{s}(t)+c_{2} \alpha_{s}^{2}(t)+o\left(\alpha_{s}^{3}(t)\right)=F\left[0, \alpha_{s}(t)\right]
$$

The content of the RGE prediction is, at this order, that there are no $\alpha_{s} t$ and $\left(\alpha_{s} t\right)^{2}$ terms (the leading log sequence must be absent) and the term of order $\alpha_{s}^{2} t$ has the coefficient that allows to reabsorb it in the transformation of $\alpha_{s}$ into $\alpha_{s}(t)$.

At present the first three coefficients have been computed in the $\overline{M S}$ scheme [15]. Clearly $c_{1}=1 / \pi$ does not depend on the definition of $\alpha_{s}$ but $c_{2}$ and $c_{3}$ do. The subleading coefficients also depend on the scale choice: if instead of expanding 
in $\alpha_{S}(Q)$ we decide to choose $\alpha_{S}(Q / 2)$ the coefficients $c_{2}$ and $c_{3}$ change. In the $\overline{M S}$ scheme, for $\gamma$-exchange and $n_{f}=5$, which are good approximations for $2 m_{b}<<$ $Q<<m_{Z}$, one has:

$$
F\left[0, \alpha_{s}(t)\right]=1+\frac{\alpha_{s}(t)}{\pi}+1.409 \ldots\left(\frac{\alpha_{s}(t)}{\pi}\right)^{2}-12.8 \ldots\left(\frac{\alpha_{s}(t)}{\pi}\right)^{3}+\ldots
$$

Similar perturbative results at 3-loop accuracy also exist for $R_{Z}=\Gamma(Z \rightarrow$ hadrons $) / \Gamma(Z \rightarrow$ leptons $), R_{\tau}=\Gamma\left(\tau \rightarrow v_{\tau}+\right.$ hadrons $) / \Gamma\left(\tau \rightarrow v_{\tau}+\right.$ leptons $)$, etc. We will discuss these results later when we deal with measurements of $\alpha_{s}$.

The perturbative expansion in powers of $\alpha_{s}(t)$ takes into account all contributions that are suppressed by powers of logarithms of the large scale $Q^{2}$ ("leading twist" terms). In addition there are corrections suppressed by powers of the large scale $Q^{2}$ ("higher twist" terms). The pattern of power corrections is controlled by the light-cone Operator Product Expansion (OPE) [16] which (schematically) leads to:

$$
F=\text { pert. }+r_{2} \frac{m^{2}}{Q^{2}}+r_{4} \frac{<0\left|\operatorname{Tr}\left[\mathbf{F}_{\mu \nu} \mathbf{F}^{\mu \nu}\right]\right| 0>}{Q^{4}}+\ldots+r_{6} \frac{<0\left|O_{6}\right| 0>}{Q^{6}}+\ldots
$$

Here $m^{2}$ generically indicates mass corrections, notably from b quarks, for example ( $\mathrm{t}$ quark mass corrections only arise from loops, vanish in the limit $m_{t} \rightarrow \infty$ and are included in the coefficients as those in Eq. (4.60) and the analogous ones for higher twist terms), $\mathbf{F}_{\mu \nu}=\sum_{A} F_{\mu \nu}^{A} t^{A}, O_{6}$ is typically a 4-fermion operator, etc. For each possible gauge invariant operator the corresponding power of $Q^{2}$ is fixed by dimensions.

We now consider the light-cone OPE in some more detail. $R_{e^{+} e^{-}} \sim \Pi\left(Q^{2}\right)$ where $\Pi\left(Q^{2}\right)$ is the scalar spectral function related to the hadronic contribution to the imaginary part of the photon vacuum polarization $T_{\mu \nu}$ :

$$
\begin{aligned}
T_{\mu \nu} & =\left(-g_{\mu \nu} Q^{2}+q_{\mu} q_{\nu}\right) \Pi\left(Q^{2}\right)=\int \exp i q x<0\left|J_{\mu}^{\dagger}(x) J_{\nu}(0)\right| 0>d x= \\
& =\sum_{n}<0\left|J_{\mu}^{\dagger}(0)\right| n><n\left|J_{\nu}(0)\right| 0>(2 \pi)^{4} \delta^{4}\left(q-p_{n}\right)
\end{aligned}
$$

For $Q^{2} \rightarrow \infty$ the $x^{2} \rightarrow 0$ region is dominant. To all orders in perturbation theory the OPE can be proven. Schematically, dropping Lorentz indices, for simplicity, near $x^{2} \sim 0$ we have:

$$
\begin{aligned}
J^{\dagger}(x) J(0)=I\left(x^{2}\right) & +E\left(x^{2}\right) \sum_{n=0}^{\infty} c_{n}\left(x^{2}\right) x^{\mu_{1}} \ldots x^{\mu_{n}} \cdot O_{\mu_{1} \ldots \mu_{n}}^{n}(0) \\
& + \text { less sing. terms }
\end{aligned}
$$


Here $I\left(x^{2}\right), E\left(x^{2}\right), \ldots, c_{n}\left(x^{2}\right)$ are c-number singular functions, $O^{n}$ is a string of local operators. $E\left(x^{2}\right)$ is the singularity of free field theory, $I\left(x^{2}\right)$ and $c_{n}\left(x^{2}\right)$ contain powers of $\log \mu^{2} x^{2}$ in interaction. Some $O^{n}$ are already present in free field theory, other ones appear when interactions are switched on. Given that $\Pi\left(Q^{2}\right)$ is related to the Fourier transform of the vacuum expectation value of the product of currents, less singular terms in $x^{2}$ lead to power suppressed terms in $1 / Q^{2}$. The perturbative terms come from $I\left(x^{2}\right)$ which is the leading twist term. The logarithmic scaling violations induced by the running coupling are the logs in $I\left(x^{2}\right)$.

\subsubsection{The Final State in $e^{+} e^{-}$Annihilation}

Experiments on $e^{+} e^{-}$annihilation at high energy provide a remarkable possibility of systematically testing the distinct signatures predicted by QCD for the structure of the final state averaged over a large number of events. Typical of asymptotic freedom is the hierarchy of configurations emerging as a consequence of the smallness of $\alpha_{s}\left(Q^{2}\right)$. When all corrections of order $\alpha_{s}\left(Q^{2}\right)$ are neglected one recovers the naive parton model prediction for the final state: almost collinear events with two backto-back jets with limited transverse momentum and an angular distribution as $(1+$ $\cos ^{2} \theta$ ) with respect to the beam axis (typical of spin $1 / 2$ parton quarks: scalar quarks would lead to a $\sin ^{2} \theta$ distribution). At order $\alpha_{S}\left(Q^{2}\right)$ a tail of events is predicted to appear with large transverse momentum $p_{T} \sim Q / 2$ with respect to the thrust axis (the axis that maximizes the sum of the absolute values of the longitudinal momenta of the final state particles). This small fraction of events with large $p_{T}$ mostly consists of three-jet events with an almost planar topology. The skeleton of a three-jet event, at leading order in $\alpha_{S}\left(Q^{2}\right)$, is formed by three hard partons $q \bar{q} g$, the third being a gluon emitted by a quark or antiquark line. The distribution of three-jet events is given by:

$$
\frac{1}{\sigma} \frac{d \sigma}{d x_{1} d x_{2}}=\frac{2 \alpha_{s}}{3 \pi} \frac{x_{1}^{2}+x_{2}^{2}}{\left(1-x_{1}\right)\left(1-x_{2}\right)}
$$

here $x_{1,2}$ refer to energy fractions of massless quarks: $x_{i}=2 E_{i} / \sqrt{s}$ with $x_{1}+x_{2}+$ $x_{3}=2$. At order $\alpha_{s}^{2}\left(Q^{2}\right)$ a hard perturbative non planar component starts to build up and a small fraction of four-jet events $q \bar{q} g g$ or $q \bar{q} q \bar{q}$ appear, and so on.

A quantitatively specified definition of jet counting must be introduced for precise QCD tests and for measuring $\alpha_{s}$, which must be infrared safe (i.e. not altered by soft particle emission or collinear splittings of massless particles) in order to be computable at parton level and as much as possible insensitive to the transformation of partons into hadrons. One introduces a resolution parameter $y_{c u t}$ and a suitable pair variable; for example [17]:

$$
y_{i j}=\frac{\min \left(E_{i}^{2}, E_{j}^{2}\right)\left(1-\cos \theta_{i j}\right)}{s}
$$


The particles i,j belong to different jets for $y_{i j}>y_{c u t}$. Clearly the number of jets becomes a function of $y_{c u t}$ : there are more jets for smaller $y_{c u t}$. Measurements of $\alpha_{s}\left(Q^{2}\right)$ have been performed starting from jet multiplicities, the largest error coming from the necessity of correcting for non-perturbative hadronisation effects.

\subsubsection{Deep Inelastic Scattering}

Deep Inelastic Scattering (DIS) processes have played and still play a very important role for our understanding of QCD and of nucleon structure. This set of processes actually provides us with a rich laboratory for theory and experiment. There are several structure functions that can be studied, $F_{i}\left(x, Q^{2}\right)$, each a function of two variables. This is true separately for different beams and targets and different polarizations. Depending on the charges of 1 and l' (see Eq. (4.13)) we can have neutral currents $(\gamma, \mathrm{Z})$ or charged currents in the 1-1' channel (Fig. 4.6). In the past DIS processes were crucial for establishing QCD as the theory of strong interactions and quarks and gluons as the QCD partons. At present DIS remains very important for quantitative studies and tests of QCD. The theory of scaling violations for totally inclusive DIS structure functions, based on operator expansion or diagrammatic techniques and renormalisation group methods, is crystal clear and the predicted $Q^{2}$ dependence can be tested at each value of $\mathrm{x}$. The measurement of quark and gluon densities in the nucleon, as functions of $\mathrm{x}$ at some reference value of $Q^{2}$, which is an essential starting point for the calculation of all relevant hadronic hard processes, is performed in DIS processes. At the same time one measures $\alpha_{S}\left(Q^{2}\right)$ and the DIS values of the running coupling can be compared with those obtained from other processes. At all times new theoretical challenges arise from the study of DIS processes. Recent examples are the so-called "spin crisis" in polarized DIS and the behaviour of singlet structure functions at small $x$ as revealed by HERA data. In the following we will review the past successes and the present open problems in the physics of DIS.

The cross-section $\sigma \sim L^{\mu \nu} W_{\mu \nu}$ is given in terms of the product of a leptonic $\left(L^{\mu \nu}\right)$ and a hadronic $\left(W_{\mu \nu}\right)$ tensor. While $L^{\mu \nu}$ is simple and easily obtained from the lowest order electroweak (EW) vertex plus QED radiative corrections, the complicated strong interaction dynamics is contained in $W_{\mu \nu}$. The latter is proportional to the Fourier transform of the forward matrix element between the nucleon target states of the product of two EW currents:

$$
W_{\mu \nu}=\int d x \exp i q x<p\left|J_{\mu}^{\dagger}(x) J_{v}(0)\right| p>
$$


Structure functions are defined starting from the general form of $W_{\mu \nu}$ given Lorentz invariance and current conservation. For example, for EW currents between unpolarized nucleons we have:

$$
\begin{aligned}
W_{\mu \nu}= & \left(-g_{\mu \nu}+\frac{q_{\mu} q_{v}}{q^{2}}\right) W_{1}\left(v, Q^{2}\right)+\left(p_{\mu}-\frac{m v}{q^{2}} q_{\mu}\right)\left(p_{\nu}-\frac{m v}{q^{2}} q_{v}\right) \frac{W_{2}\left(v, Q^{2}\right)}{m^{2}}- \\
& -\frac{i}{2 m^{2}} \epsilon_{\mu \nu \lambda \rho} p^{\lambda} q^{\rho} W_{3}\left(v, Q^{2}\right)
\end{aligned}
$$

$W_{3}$ arises from VA interference and is absent for pure vector currents. In the limit $Q^{2}>>m^{2}$, x fixed, the structure functions obey approximate Bjorken scaling which in reality is broken by logarithmic corrections that can be computed in QCD:

$$
\begin{aligned}
m W_{1}\left(\nu, Q^{2}\right) & \rightarrow F_{1}(x) \\
\nu W_{2,3}\left(\nu, Q^{2}\right) & \rightarrow F_{2,3}(x)
\end{aligned}
$$

The $\gamma-N$ cross-section is given by $\left(W_{i}=W_{i}\left(Q^{2}, v\right)\right)$ :

$$
\frac{d \sigma^{\gamma}}{d Q^{2} d \nu}=\frac{4 \pi \alpha^{2} E^{\prime}}{Q^{4} E} \cdot\left[2 \sin ^{2} \frac{\theta}{2} W_{1}+\cos ^{2} \frac{\theta}{2} W_{2}\right]
$$

while for the $v-N$ or $\bar{v}-N$ cross-section one has:

$$
\frac{d \sigma^{\nu, \bar{v}}}{d Q^{2} d \nu}=\frac{G_{F}^{2} E^{\prime}}{2 \pi E}\left(\frac{m_{W}^{2}}{Q^{2}+m_{W}^{2}}\right)^{2} \cdot\left[2 \sin ^{2} \frac{\theta}{2} W_{1}+\cos ^{2} \frac{\theta}{2} W_{2} \pm \frac{E+E^{\prime}}{m} \sin ^{2} \frac{\theta}{2} W_{3}\right]
$$

( $W_{i}$ for photons, $v$ and $\bar{v}$ are all different, as we shall see in a moment).

In the scaling limit the longitudinal and transverse cross sections are given by:

$$
\begin{aligned}
\sigma_{L} & \sim \frac{1}{s}\left[\frac{F_{2}(x)}{2 x}-F_{1}(x)\right] \\
\sigma_{R H, L H} & \sim \frac{1}{s}\left[F_{1}(x) \pm F_{3}(x)\right] \\
\sigma_{T} & =\sigma_{R H}+\sigma_{L H}
\end{aligned}
$$

where $\mathrm{L}, \mathrm{RH}, \mathrm{LH}$ refer to the helicity $0,1,-1$, respectively, of the exchanged gauge vector boson.

In the '60's the demise of hadrons from the status of fundamental particles to that of bound states of constituent quarks was the breakthrough that made possible the construction of a renormalisable field theory for strong interactions. The presence of an unlimited number of hadrons species, many of them with large spin values, presented an obvious dead-end for a manageable field theory. The evidence for constituent quarks emerged clearly from the systematics of hadron spectroscopy. 
The complications of the hadron spectrum could be explained in terms of the quantum numbers of spin $1 / 2$, fractionally charged, $u, d$ and s quarks. The notion of colour was introduced to reconcile the observed spectrum with Fermi statistics. But confinement that forbids the observation of free quarks was a clear obstacle towards the acceptance of quarks as real constituents and not just as fictitious entities describing some mathematical pattern (a doubt expressed even by GellMann at the time). The early measurements at SLAC of DIS dissipated all doubts: the observation of Bjorken scaling and the success of the "naive" (not so much after all) parton model of Feynman imposed quarks as the basic fields for describing the nucleon structure (parton quarks).

In the language of Bjorken and Feynman the virtual $\gamma$ (or, in general, any gauge boson) sees the quark partons inside the nucleon target as quasi-free, because their (Lorentz dilated) QCD interaction time is much longer than $\tau_{\gamma} \sim 1 / Q$, the duration of the virtual photon interaction. Since the virtual photon 4-momentum is spacelike, we can go to a Lorentz frame where $E_{\gamma}=0$ (Breit frame). In this frame $q=\left(E_{\gamma}=0 ; 0,0, Q\right)$ and the nucleon momentum, neglecting the mass $m<<Q$, is $p=(Q / 2 x ; 0,0,-Q / 2 x)$ (note that this correctly gives $q^{2}=-Q^{2}$ and $x=Q^{2} / 2(p \cdot q)$ ). Consider (Fig. 4.10) the interaction of the photon with a quark carrying a fraction y of the nucleon 4-momentum: $p_{q}=y p$ (we are neglecting the transverse components of $p_{q}$ which are of order $m$ ). The incoming parton with $p_{q}=y p$ absorbs the photon and the final parton has 4-momentum $p_{q}^{\prime}$. Since in the Breit frame the photon carries no energy but only a longitudinal momentum $Q$, the photon can only be absorbed by those partons with $y=x$ : then the longitudinal component of $p_{q}=y p$ is $-y Q / 2 x=-Q / 2$ and can be flipped into $+Q / 2$ by the photon. As a result, the photon longitudinal momentum $+Q$ disappears, the parton quark momentum changes of sign from $-Q / 2$ into $+Q / 2$ and the energy is not changed. So the structure functions are proportional to the density of partons with fraction $\mathrm{x}$ of the nucleon momentum, weighted with the squared charge. Also, recall that the helicity of a massless quark is conserved in a vector (or axial vector) interaction. So when the momentum is reversed also the spin must flip. Since the process is collinear there is no orbital contribution and only a photon with helicity \pm 1 (transverse photon) can be absorbed. Alternatively, if partons were spin zero only longitudinal photons would instead contribute.

Using these results, which are maintained in QCD at leading order, the quantum numbers of the quarks were confirmed by early experiments. The observation that $R=\sigma_{L} / \sigma_{T} \rightarrow 0$ implies that the charged partons have spin $1 / 2$. The quark charges

Fig. 4.10 Schematic diagram for the interaction of the virtual photon with a parton quark in the Breit frame

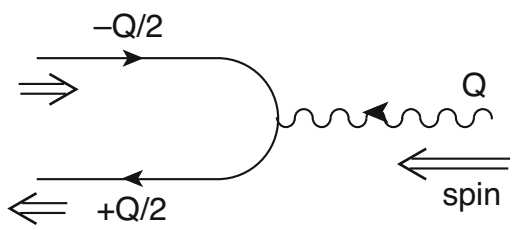


were derived from the data on the electron and neutrino structure functions:

$$
\begin{aligned}
& F_{e p}=4 / 9 u(x)+1 / 9 d(x)+\ldots \ldots ; \quad F_{e n}=4 / 9 d(x)+1 / 9 u(x)+\ldots \\
& F_{v p}=F_{\bar{v} n}=2 d(x)+\ldots \ldots ; \quad F_{v n}=F_{\bar{v} p}=2 u(x)+\ldots \ldots
\end{aligned}
$$

where $F \sim 2 F_{1} \sim F_{2} / x$ and $u(x), d(x)$ are the parton number densities in the proton (with fraction $\mathrm{x}$ of the proton longitudinal momentum), which, in the scaling limit, do not depend on $Q^{2}$. The normalisation of the structure functions and the parton densities are such that the charge relations hold:

$$
\int_{0}^{1}[u(x)-\bar{u}(x)] d x=2, \quad \int_{0}^{1}[d(x)-\bar{d}(x)] d x=1, \quad \int_{0}^{1}[s(x)-\bar{s}(x)] d x=0
$$

Also it was proven by experiment that at values of $Q^{2}$ of a few $\mathrm{GeV}^{2}$, in the scaling region, about half of the nucleon momentum, given by the momentum sum rule:

$$
\int_{0}^{1}\left[\sum_{i}\left(q_{i}(x)+\bar{q}_{i}(x)\right)+g(x)\right] x d x=1
$$

is carried by neutral partons (gluons).

In QCD there are calculable log scaling violations induced by $\alpha_{s}(t)$. The parton rules just introduced can be summarised in the formula:

$$
F(x, t)=\int_{x}^{1} d y \frac{q_{0}(y)}{y} \sigma_{p o i n t}\left(\frac{x}{y}, \alpha_{s}(t)\right)+o\left(\frac{1}{Q^{2}}\right)
$$

Before QCD corrections $\sigma_{\text {point }}=e^{2} \delta(x / y-1)$ and $F=e^{2} q_{0}(x)$ (here we denote by $e$ the charge of the quark in units of the positron charge, i.e. $e=2 / 3$ for the $u$ quark). QCD modifies $\sigma_{\text {point }}$ at order $\alpha_{s}$ via the diagrams of Fig. 4.11. Note that the integral is from $\mathrm{x}$ to 1 , because the energy can only be lost by radiation before interacting with the photon (which eventually wants to find a fraction $x$, as we have

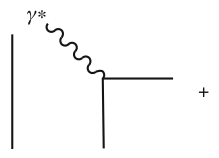

(a)

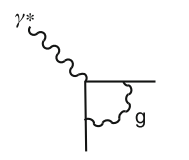

(b)

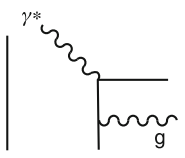

(c)

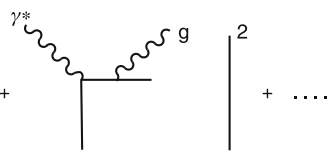

(d)

Fig. 4.11 First order QCD corrections to the virtual photon-quark cross-section: (a) leading order with (b) one-loop virtual correction; (c-d) next-to-leading order real emission 
explained). From a direct computation of the diagrams one obtains a result of the following form:

$$
\sigma_{\text {point }}\left(z, \alpha_{s}(t)\right) \simeq e^{2}\left[\delta(z-1)+\frac{\alpha_{s}}{2 \pi}(t \cdot P(z)+f(z))\right]
$$

For $y>x$ the correction arises from diagrams with real gluon emission. Only the sum of the two real diagrams in Fig. 4.11 is gauge invariant, so that the contribution of one given diagram is gauge dependent. There is a special form of axial gauge, called physical gauge, where, among real diagrams, the diagram of Fig. $4.11 \mathrm{c}$ gives the whole $t$-proportional term. It is obviously not essential to go to this gauge, but this diagram has a direct physical interpretation: a quark in the proton has a fraction $y>x$ of the parent 4-momentum; it then radiates a gluon and looses energy down to a fraction $x$ before interacting with the photon. The log arises from the virtual quark propagator, according to the discussion of collinear mass singularities in Eq. (4.12). In fact in the massless limit one has:

$$
\begin{aligned}
\text { propagator } & =\frac{1}{r^{2}}=\frac{1}{(k-h)^{2}}=\frac{-1}{2 E_{k} E_{h}} \cdot \frac{1}{1-\cos \theta} \\
& =\frac{-1}{4 E_{k} E_{h}} \cdot \frac{1}{\sin ^{2} \theta / 2} \propto \frac{-1}{p_{T}^{2}}
\end{aligned}
$$

where $p_{T}$ is the transverse momentum of the virtual quark. So the square of the propagator goes like $1 / p_{T}^{4}$. But there is a $p_{T}^{2}$ factor in the numerator, because in the collinear limit, when $\theta=0$ and the initial and final quarks and the emitted gluon are all aligned, the quark helicity cannot flip (vector interaction) so that the gluon should carry helicity zero but a real gluon can only have \pm 1 helicity. Thus the numerator vanishes as $p_{T}^{2}$ in the forward direction and the cross-section behaves as:

$$
\sigma \sim \int^{Q^{2}} \frac{1}{p_{T}^{2}} d p_{T}^{2} \sim \log Q^{2}
$$

Actually the $\log$ should be read as $\log Q^{2} / \mathrm{m}^{2}$ because in the massless limit a genuine mass singularity appears. In fact the mass singularity connected with the initial quark line is not cancelled because we do not have the sum of all degenerate initial states, but only a single quark. But in correspondence to the initial quark we have the (bare) quark density $q_{0}(y)$ that appear in the convolution integral. This is a non perturbative quantity that is determined by the nucleon wave function. So we can factorize the mass singularity in a redefinition of the quark density: we replace $q_{0}(y) \rightarrow q(y, t)=q_{0}(y)+\Delta q(y, t)$ with:

$$
\Delta q(x, t)=\frac{\alpha_{s}}{2 \pi} t \int_{x}^{1} d y \frac{q_{0}(y)}{y} \cdot P\left(\frac{x}{y}\right)
$$


Here the factor of $t$ is a bit symbolic: it stands for $\log Q^{2} / \mathrm{km}^{2}$ and what we exactly put below $Q^{2}$ depends on the definition of the renormalised quark density, which also fixes the exact form of the finite term $f(z)$ in Eq. (4.75).

The effective parton density $q(y, t)$ that we have defined is now scale dependent. In terms of this scale dependent density we have the following relations, where we have also replaced the fixed coupling with the running coupling according to the prescription derived from the RGE:

$$
\begin{aligned}
F(x, t) & \left.=\int_{x}^{1} d y \frac{q(y, t)}{y} e^{2}\left[\delta\left(\frac{x}{y}-1\right)+\frac{\alpha_{s}(t)}{2 \pi} f\left(\frac{x}{y}\right)\right)\right]=e^{2} q(x, t)+o\left(\alpha_{s}(t)\right) \\
\frac{d}{d t} q(x, t) & =\frac{\alpha_{s}(t)}{2 \pi} \int_{x}^{1} d y \frac{q(y, t)}{y} \cdot P\left(\frac{x}{y}\right)+o\left(\alpha_{s}(t)^{2}\right)
\end{aligned}
$$

We see that in lowest order we reproduce the naive parton model formulae for the structure functions in terms of effective parton densities that are scale dependent. The evolution equations for the parton densities are written down in terms of kernels (the "splitting functions") that can be expanded in powers of the running coupling. At leading order, we can interpret the evolution equation by saying that the variation of the quark density at $x$ is given by the convolution of the quark density at $y$ times the probability of emitting a gluon with fraction $x / y$ of the quark momentum.

It is interesting that the integro-differential QCD evolution equation for densities can be transformed into an infinite set of ordinary differential equations for Mellin moments [2]. The moment $f_{n}$ of a density $f(x)$ is defined as:

$$
f_{n}=\int_{0}^{1} d x x^{n-1} f(x)
$$

By taking moments of both sides of the second of Eqs. (4.79) one finds, with a simple interchange of the integration order, the simpler equation for the $n$-th moment:

$$
\frac{d}{d t} q_{n}(t)=\frac{\alpha_{s}(t)}{2 \pi} \cdot P_{n} \cdot q_{n}(t)
$$

To solve this equation we observe that:

$$
\log \frac{q_{n}(t)}{q_{n}(0)}=\frac{P_{n}}{2 \pi} \int_{0}^{t} \alpha_{s}(t) d t=\frac{P_{n}}{2 \pi} \int_{\alpha_{s}}^{\alpha_{s}(t)} \frac{d \alpha^{\prime}}{-b \alpha^{\prime}}
$$

where we used Eq. (4.31) to change the integration variable from $d t$ to $d \alpha(t)$ (denoted as $d \alpha^{\prime}$ ) and $\beta(\alpha) \simeq-b \alpha^{2}+\ldots$. Finally the solution is:

$$
q_{n}(t)=\left[\frac{\alpha_{s}}{\alpha_{s}(t)}\right]^{\frac{P_{n}}{2 \pi b}} \cdot q_{n}(0)
$$


The connection of these results with the RGE general formalism occurs via the light cone OPE (recall Eq. (4.66) for $W_{\mu \nu}$ and Eq. (4.63) for the OPE of two currents). In the case of DIS the c-number term $I\left(x^{2}\right)$ does not contribute, because we are interested in the connected part $<p|\ldots| p>-<0|\ldots| 0>$. The relevant terms are:

$$
J^{\dagger}(x) J(0)=E\left(x^{2}\right) \sum_{n=0}^{\infty} c_{n}\left(x^{2}\right) x^{\mu_{1}} \ldots x^{\mu_{n}} \cdot O_{\mu_{1} \ldots \mu_{n}}^{n}(0)+\text { less sing. terms }
$$

A formally intricate but conceptually simple argument (Ref. [6], page 28) based on the analiticity properties of the forward virtual Compton amplitude shows that the Mellin moments $M_{n}$ of structure functions are related to the individual terms in the OPE, precisely to the Fourier transform $c_{n}\left(Q^{2}\right)$ (we will write it as $c_{n}(t, \alpha)$ ) of the coefficient $c_{n}\left(x^{2}\right)$ times a reduced matrix element $h_{n}$ from the operators $O^{n}$ : $<p\left|O_{\mu_{1} \ldots \mu_{n}}^{n}(0)\right| p>=h_{n} p_{\mu_{1}} \ldots p_{\mu_{n}}$ :

$$
c_{n}<p\left|O^{n}\right| p>\rightarrow M_{n}=\int_{0}^{1} d x x^{n-1} F(x)
$$

Since the matrix element of the products of currents satisfy the RGE so do the moments $M_{n}$. Hence the general form of the $Q^{2}$ dependence is given by the RGE solution (see Eq. (4.33)):

$$
M_{n}(t, \alpha)=c_{n}[0, \alpha(t)] \exp \int_{\alpha}^{\alpha(t)} \frac{\gamma_{n}\left(\alpha^{\prime}\right)}{\beta\left(\alpha^{\prime}\right)} d \alpha^{\prime} \cdot h_{n}(\alpha)
$$

In lowest order, identifying in the simplest case $M_{n}$ with $q_{n}$, we have:

$$
\gamma_{n}(\alpha)=\frac{P_{n}}{2 \pi} \alpha+\ldots, \quad \beta(\alpha)=-b \alpha^{2}+\ldots
$$

and

$$
q_{n}(t)=q_{n}(0) \exp \int_{\alpha}^{\alpha(t)} \frac{\gamma_{n}\left(\alpha^{\prime}\right)}{\beta\left(\alpha^{\prime}\right)} d \alpha^{\prime}=\left[\frac{\alpha_{s}}{\alpha_{S}(t)}\right]^{\frac{P_{n}}{2 \pi b}} \cdot q_{n}(0)
$$

which exactly coincides with Eq. (4.83).

Up to this point we have implicitly restricted our attention to non-singlet (under the flavour group) structure functions. The $Q^{2}$ evolution equations become non diagonal as soon as we take into account the presence of gluons in the target. In fact the quark which is seen by the photon can be generated by a gluon in the target (Fig. 4.12). 
Fig. 4.12 Lowest order diagram for the interaction of the virtual photon with a parton gluon

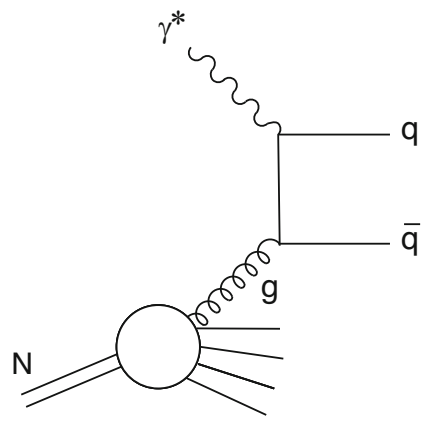

The quark evolution equation becomes:

$$
\frac{d}{d t} q_{i}(x, t)=\frac{\alpha_{s}(t)}{2 \pi}\left[q_{i} \otimes P_{q q}\right]+\frac{\alpha_{s}(t)}{2 \pi}\left[g \otimes P_{q g}\right]
$$

where we introduced the shorthand notation:

$$
[q \otimes P]=[P \otimes q]=\int_{x}^{1} d y \frac{q(y, t)}{y} \cdot P\left(\frac{x}{y}\right)
$$

(it is easy to check that the convolution, like an ordinary product, is commutative). At leading order, the interpretation of Eq. (4.89) is simply that the variation of the quark density is due to the convolution of the quark density at a higher energy times the probability of finding a quark in a quark (with the right energy fraction) plus the gluon density at a higher energy times the probability of finding a quark (of the given flavour i) in a gluon. The evolution equation for the gluon density, needed to close the system, can be obtained by suitably extending the same line of reasoning to a gedanken probe sensitive to colour charges, for example a virtual gluon. The resulting equation is of the form:

$$
\frac{d}{d t} g(x, t)=\frac{\alpha_{s}(t)}{2 \pi}\left[\sum_{i}\left(q_{i}+\bar{q}_{i}\right) \otimes P_{g q}\right]+\frac{\alpha_{s}(t)}{2 \pi}\left[g \otimes P_{g g}\right]
$$

The explicit form of the splitting functions in lowest order [18, 19] can be directly derived from the QCD vertices [19]. They are a property of the theory and do not depend on the particular process the parton density is taking part in. The results are:

$$
\begin{aligned}
& P_{q q}=\frac{4}{3}\left[\frac{1+x^{2}}{(1-x)_{+}}+\frac{3}{2} \delta(1-x)\right]+o\left(\alpha_{s}\right) \\
& P_{g q}=\frac{4}{3} \frac{1+(1-x)^{2}}{x}+o\left(\alpha_{s}\right)
\end{aligned}
$$




$$
\begin{aligned}
& P_{q g}=\frac{1}{2}\left[x^{2}+(1-x)^{2}\right]+o\left(\alpha_{s}\right) \\
& P_{g g}=6\left[\frac{x}{(1-x)_{+}}+\frac{1-x}{x}+x(1-x)\right]+\frac{33-2 n_{f}}{6} \delta(1-x)+o\left(\alpha_{s}\right)
\end{aligned}
$$

For a generic non singular weight function $f(x)$, the " + " distribution is defined as:

$$
\int_{0}^{1} \frac{f(x)}{(1-x)_{+}} d x=\int_{0}^{1} \frac{f(x)-f(1)}{1-x} d x
$$

The $\delta(1-x)$ terms arise from the virtual corrections to the lowest order tree diagrams. Their coefficient can be simply obtained by imposing the validity of charge and momentum sum rules. In fact, from the request that the charge sum rules in Eq. (4.72) are not affected by the $Q^{2}$ dependence one derives that

$$
\int_{0}^{1} P_{q q}(x) d x=0
$$

which can be used to fix the coefficient of the $\delta(1-x)$ terms of $P_{q q}$. Similarly, by taking the t-derivative of the momentum sum rule in Eq. (4.73) and imposing its vanishing for generic $q_{i}$ and $g$, one obtains:

$$
\int_{0}^{1}\left[P_{q q}(x)+P_{g q}(x)\right] x d x=0, \quad \int_{0}^{1}\left[2 n_{f} P_{q g}(x)+P_{g g}(x)\right] x d x=0 .
$$

At higher orders the evolution equations are easily generalised but the calculation of the splitting functions rapidly becomes very complicated. For many years the splitting functions were only completely known at NLO accuracy [20]: $\alpha_{s} P \sim \alpha_{s} P_{1}+\alpha_{s}^{2} P_{2}+\ldots$. Then in recent years the NNLO results $P_{3}$ have been first derived in analytic form for the first few moments and, then the full NNLO analytic calculation, a really monumental work, was completed in 2004 by Moch, Vermaseren and Vogt [21]. Beyond leading order a precise definition of parton densities should be specified. One can take a physical definition (for example, quark densities can be defined as to keep the LO expression for the structure function $F_{2}$ valid at all orders, the so called DIS definition [22], and the gluon density could be defined starting from $F_{L}$, the longitudinal structure function, or a more abstract specification (for example, in terms of the $\overline{M S}$ prescription). Once the definition of parton densities is fixed, the coefficients that relate the different structure functions to the parton densities at each fixed order can be computed. Similarly the higher order splitting functions also depend, to some extent, from the definition of parton densities, and a consistent set of coefficients and splitting functions must be used at each order.

The scaling violations are clearly observed by experiment and their pattern is very well reproduced by QCD fits at NLO. Examples are seen in Fig. 4.13a-d [23]. 

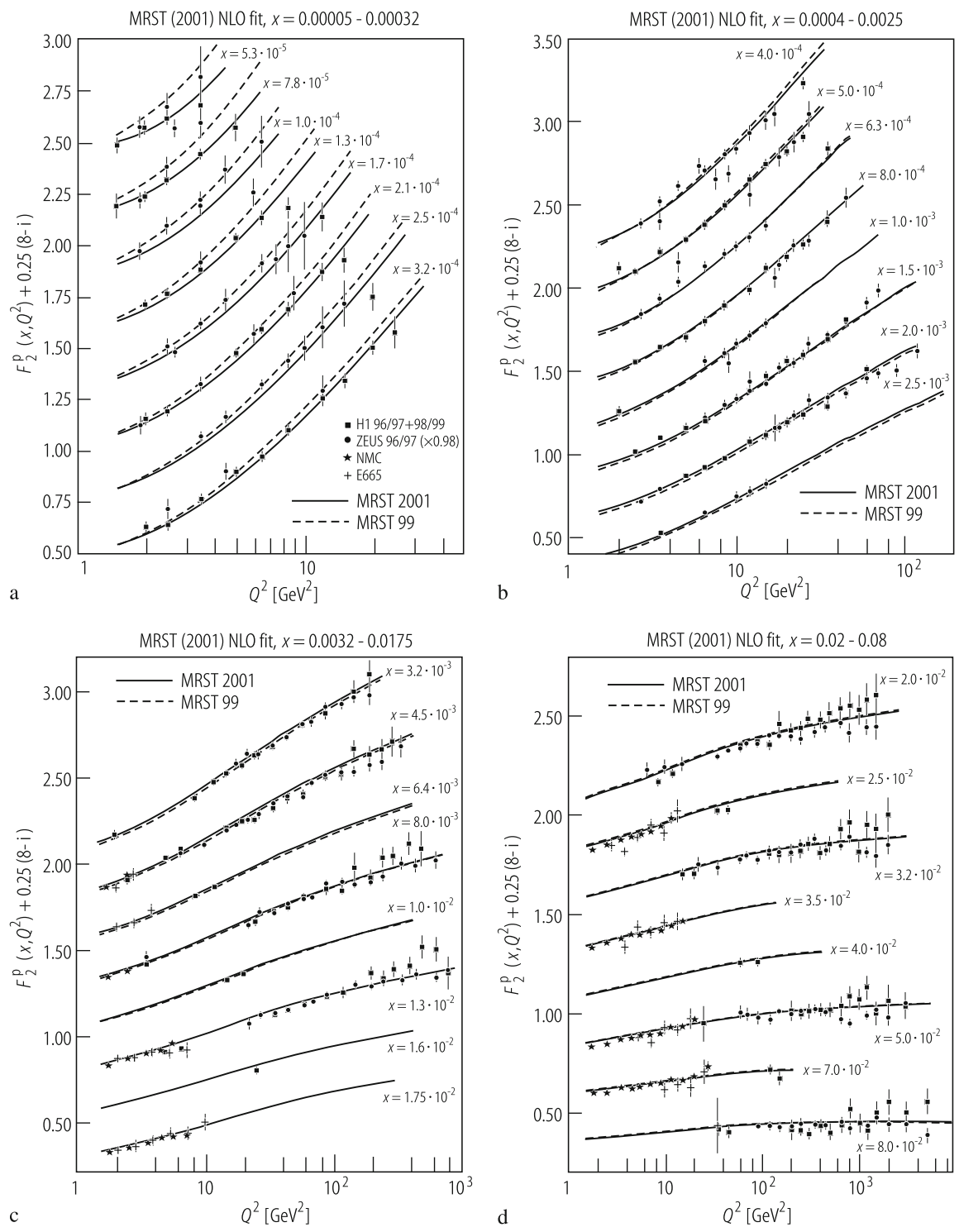

Fig. 4.13 A recent NLO fit of scaling violations from Ref. [23], for different $x$ ranges, as functions of $Q^{2}$ 
These fits provide an impressive confirmation of a quantitative QCD prediction, a measurement of $q_{i}\left(x, Q_{0}^{2}\right)$ and $g\left(x, Q_{0}^{2}\right)$ at some reference value $Q_{0}^{2}$ of $Q^{2}$ and a precise measurement of $\alpha_{s}\left(m_{Z}^{2}\right)$.

\subsubsection{Resummation for Deep Inelastic Structure Functions}

At small or at large values of $x$ (with $Q^{2}$ large) those terms of higher order in $\alpha_{s}$ in either the coefficients or the splitting functions which are multiplied by powers of $\log 1 / x$ or $\log (1-x)$ eventually become important and should be taken into account. Fortunately the sequences of leading and subleading logs can be evaluated at all orders by special techniques and resummed to all orders.

For large x resummation [24] I refer to the recent papers [25, 26] (the latter also involving higher twist corrections, which are important at large $\mathrm{x}$ ) where a list of references to previous work can be found.

Here we will briefly summarise the small-x case for the singlet structure function which is the dominant channel at HERA, dominated by the sharp rise of the gluon and sea parton densities at small $\mathrm{x}$. The small $x$ data collected by HERA can be fitted reasonably well even at the smallest measured values of $x$ by the NLO QCD evolution equations, so that there is no dramatic evidence in the data for departures. This is surprising also in view of the fact that the NNLO effects in the evolution have recently become available and are quite large. Resummation effects have been shown to resolve this apparent paradox. For the singlet splitting function the coefficients of all LO and NLO corrections of order $\left[\alpha_{s}\left(Q^{2}\right) \log 1 / x\right]^{n}$ and $\alpha_{s}\left(Q^{2}\right)\left[\alpha_{s}\left(Q^{2}\right) \log 1 / x\right]^{n}$, respectively, are explicitly known from the BFKL analysis of virtual gluon-virtual gluon scattering [27, 28]. But the simple addition of these higher order terms to the perturbative result (with subtraction of all double counting) does not lead to a converging expansion (the NLO logs completely overrule the LO $\operatorname{logs}$ in the relevant domain of $x$ and $Q^{2}$ ). A sensible expansion is only obtained by a proper treatment of momentum conservation constraints, also using the underlying symmetry of the BFKL kernel under exchange of the two external gluons, and especially, of the running coupling effects (see the recent papers $[29,30]$ and refs. therein). In Fig. 4.14 we present the results for the dominant singlet splitting function $x P\left(x, \alpha_{s}\left(Q^{2}\right)\right)$ for $\alpha_{s}\left(Q^{2}\right) \sim 0.2$. We see that while the NNLO perturbative splitting function sharply deviates from the NLO approximation at small $x$, the resummed result only shows a moderate dip with respect to the NLO perturbative splitting function in the region of HERA data, and the full effect of the true small $\mathrm{x}$ asymptotics is only felt at much smaller values of $\mathrm{x}$. The related effects are not very important for processes at the LHC but could become relevant for next generation hadron colliders. 
Fig. 4.14 The dominant singlet splitting function $x P\left(x, \alpha_{s}\left(Q^{2}\right)\right)$ for $\alpha_{s}\left(Q^{2}\right) \sim 0.2$. The resummed result from Ref. [29] is compared with the LO, NLO and NNLO perturbative results

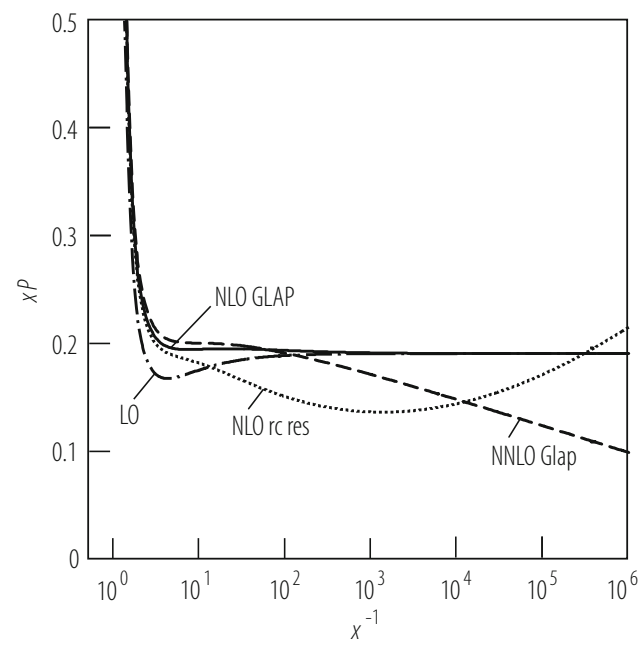

\subsubsection{Polarized Deep Inelastic Scattering}

In polarized DIS one main question is how the proton helicity is distributed among quarks, gluons and orbital angular momentum: $1 / 2 \Delta \Sigma+\Delta g+L_{z}=1 / 2$ (for a recent review, see, for example, [31]). For a parton density $p$ (either a quark or a gluon) $\Delta p$ indicates the first moment of the net helicity difference $p_{+}-p_{-}$in a polarized proton with helicity $+1 / 2$ or:

$$
\Delta p\left(Q^{2}\right)=\int_{0}^{1} d x\left[p_{+}\left(x, Q^{2}\right)-p_{-}\left(x, Q^{2}\right)\right]
$$

Experiments have shown that the quark moment $\Delta \Sigma$ is small (the "spin crisis"): values from a recent fit [32] are $\Delta \Sigma_{\text {exp }} \sim 0.21 \pm 0.14$ and $\Delta g_{\text {exp }} \sim 0.50 \pm 1.27$ at $Q^{2}=1 \mathrm{GeV}^{2}$ (see also [33]). This is surprising because $\Delta \Sigma$ is conserved in perturbation theory at LO (i.e. it does not evolve in $Q^{2}$ ). For conserved quantities we would expect that they are the same for constituent and for parton quarks. But actually the conservation of $\Delta \Sigma$ is broken by the axial anomaly. In perturbation theory the conserved density is actually $\Delta \Sigma^{\prime}=\Delta \Sigma+n_{f} / 2 \pi \alpha_{s} \Delta g$ [34]. Note that also $\alpha_{s} \Delta g$ is conserved in LO, that is $\Delta g \sim \log Q^{2}$. This behaviour is not controversial but it will take long before the log growth of $\Delta g$ will be confirmed by experiment! But to establish this behaviour would show that the extraction of $\Delta g$ from the data is correct and that the QCD evolution works as expected. If $\Delta g$ is large enough it could account for the difference between partons $(\Delta \Sigma)$ and constituents ( $\left.\Delta \Sigma^{\prime}\right)$. From the spin sum rule it is clear that the log increase should cancel between $\Delta g$ and $L_{z}$. This cancelation is automatic as a consequence of helicity conservation in the basic QCD vertices. From the spin sum rule one obtains that either $\Delta g+L_{z}$ is large or there are contributions to $\Delta \Sigma$ at very small $\mathrm{x}$ outside of the measured region. 
$\Delta g$ can be measured indirectly by scaling violations and directly from asymmetries, e.g. in $c \bar{c}$ production. Existing measurements by Hermes, Compass, and at RHIC are still crude but show no hint of a large $\Delta g$ at accessible value of $x$ and $Q^{2}$. Present data are consistent with $\Delta g$ large enough to sizeably contribute to the spin sum rule but there is no indication that $\alpha_{s} \Delta g$ can explain the difference between constituents and parton quarks. The perspectives of better measurements are good at Compass and RHIC in the near future.

\subsubsection{Factorisation and the QCD Improved Parton Model}

The parton densities defined and measured in DIS are instrumental to compute hard processes initiated by hadronic collisions via the Factorisation Theorem (FT). Suppose you have a hadronic process of the form $h_{1}+h_{2} \rightarrow X+$ all where $h_{i}$ are hadrons and $X$ is some triggering particle or pair of particles which specify the large scale $Q^{2}$ relevant for the process, in general somewhat, but not much, smaller than $\mathrm{s}$, the total c.o.m. squared mass. For example, in $p p$ or $p \bar{p}$ collisions, $X$ can be a $W$ or a $\mathrm{Z}$ or a virtual photon with large $Q^{2}$, or a jet at large transverse momentum $p_{T}$, or a pair of heavy quark-antiquark of mass M. By "all” we mean a totally inclusive collection of gluons and light quark pairs. The FT states that for the total crosssection or some other sufficiently inclusive distribution we can write, apart from power suppressed corrections, the expression:

$$
\sigma(s, \tau)=\sum_{A B} \int d x_{1} d x_{2} p_{1 A}\left(x_{1}, Q^{2}\right) p_{2 B}\left(x_{2}, Q^{2}\right) \sigma_{A B}\left(x_{1} x_{2} s, \tau\right)
$$

Here $\tau=Q^{2} / s$ is a scaling variable, $p_{i C}$ are the densities for a parton of type $\mathrm{C}$ inside the hadron $h_{i}, \sigma_{A B}$ is the partonic cross-section for parton-A + parton$\mathrm{B} \rightarrow X+a l l^{\prime}$. This result is based on the fact that the mass singularities that are associated with the initial legs are of universal nature, so that one can reproduce the same modified parton densities, by absorbing these singularities into the bare parton densities, as in deep inelastic scattering. Once the parton densities and $\alpha_{s}$ are known from other measurements, the prediction of the rate for a given hard process is obtained with not much ambiguity (e.g from scale dependence or hadronisation effects). The NLO calculation of the reduced partonic cross-section is needed in order to correctly specify the scale and in general the definition of the parton densities and of the running coupling in the leading term. The residual scale and scheme dependence is often the most important source of theoretical error. In the following we consider a few examples.

A comparison of data and predictions on the production of jets at large $\sqrt{s}$ and $p_{T}$ in $p p$ or $p \bar{p}$ collisions is shown in Fig. 4.15 [9, 35].

This is a particularly significant test because the rates at different c.o.m. energies and, for each energy, at different values of $p_{T}$ span over many orders of magnitude. 
Fig. 4.15 Jet production cross-section at $p p$ or $p \bar{p}$ colliders, as function of $p_{T}[9]$

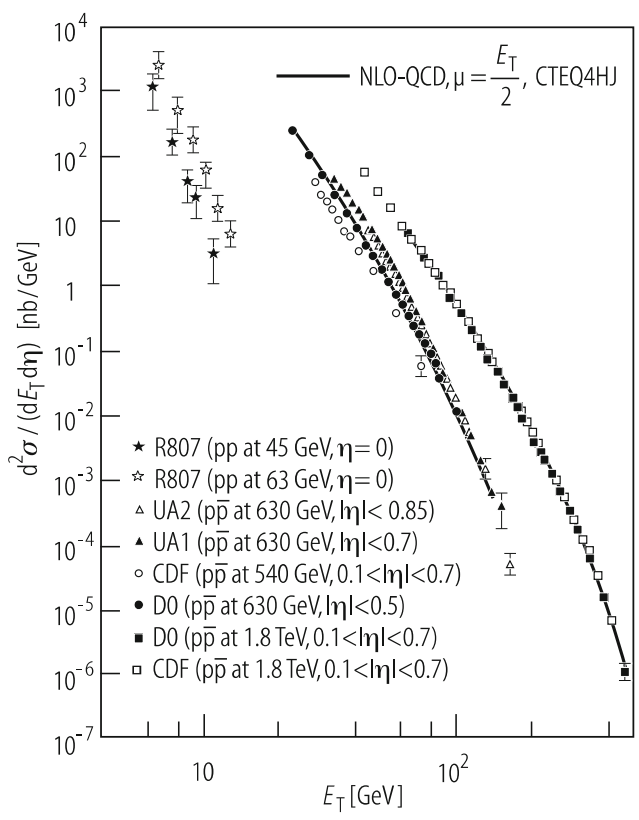

This steep behaviour is determined by the sharp falling of the parton densities at large $\mathrm{x}$. Also the corresponding values of $\sqrt{s}$ and $p_{T}$ are large enough to be well inside the perturbative region. The overall agreement of the data from ISR, UA1,2 and CDF and D0 is spectacular. Similar results also hold for the production of photons at large $p_{T}$. The collider data [36], shown in Fig. 4.16 [9], are in fair agreement with the theoretical predictions. For the same process less clear a situation is found with fixed target data. Here, first of all, the experimental results show some internal discrepancies. Also, the $p_{T}$ accessible values being smaller, the theoretical uncertainties are larger. But it is true that the agreement is poor, so that the necessity of an "intrinsic" transverse momentum of partons inside the hadron of over $1 \mathrm{GeV}$ has been claimed, which theoretically is not justified (rather, given the sharp falling down at large $p_{T}$, it could be interpreted as a correction for $p_{T}$ calibration errors).

For heavy quark production at colliders [42] the agreement is very good for the top crosssection at the Tevatron (Fig. 4.17) [43, 44]. The bottom production at the Tevatron has for some time represented a problem [45]. The total rate and the $p_{T}$ distribution of $b$ quarks observed at CDF appeared in excess of the prediction, up to the largest measured values of $p_{T}$. But this is a complicated case, with different scales being present at the same time: $\sqrt{s}, p_{T}, m_{b}$. Finally the problem has been solved (Fig. 4.18) by better taking into account a number of small effects from resummation of large logarithms, the difference between $b$ hadrons and $b$ partons, the inclusion of better fragmentation functions etc. [46]. 
Fig. 4.16 Single photon production in $p \bar{p}$ colliders as function of $p_{T}[9]$

Fig. 4.17 The $t$ production cross-section at the Tevatron $p \bar{p}$ collider [44]
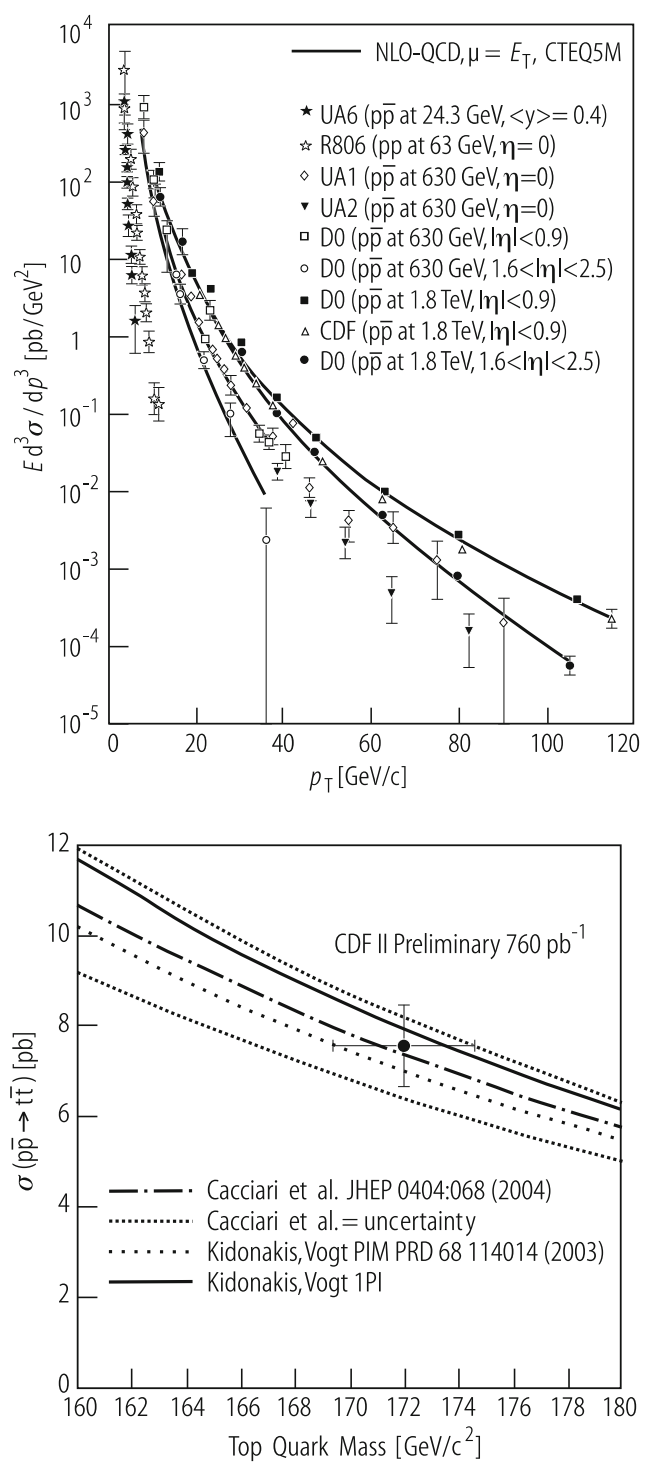

Drell-Yan processes, including lepton pair production via virtual $\gamma, \mathrm{W}$ or $\mathrm{Z}$ exchange, offer a particularly good opportunity to test QCD. The process is quadratic in parton densities, and the final state is totally inclusive, while the large scale is specified and measured by the invariant mass squared $Q^{2}$ of the lepton pair which itself is not strongly interacting (so there no dangerous hadronisation effects). The QCD improved parton model leads directly to a prediction for the total rate as a function of $Q^{2}$. The value of the LO cross-section is inversely proportional to the number of colours $N_{C}$ because a quark of given colour can only annihilate with an 
Fig. 4.18 The $b$ production $p_{T}$ distribution at the Tevatron $p \bar{p}$ collider [47]. The data from CDF also include systematics and correlations. The theoretical curve with the uncertainty range is from Ref. [46]

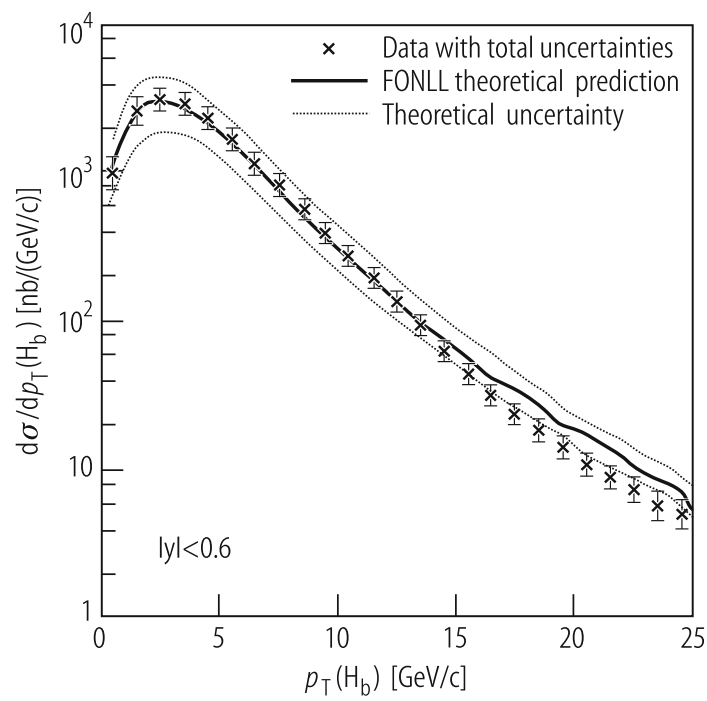

antiquark of the same colour to produce a colourless lepton pair. The order $\alpha_{S}\left(Q^{2}\right)$ corrections to the total rate were computed long ago and found to be particularly large $[22,38]$, when the quark densities are defined from the structure function $F_{2}$ measured in DIS at $q^{2}=-Q^{2}$. The ratio $\sigma_{c o r r} / \sigma_{L O}$ of the corrected and the Born cross-sections, was called $\mathrm{K}$-factor, because it is almost a constant in rapidity. In recent years also the NLO full calculation of the K-factor was completed, a very remarkable calculation [37]. The QCD predictions have been best tested for $\mathrm{W}$ and $\mathrm{Z}$ production at CERN $\operatorname{Sp} \bar{p} S$ and Tevatron energies. $Q \sim m_{W, Z}$ is large enough to make the prediction reliable (with a not too large K-factor) and the ratio $\sqrt{\tau}=Q / \sqrt{s}$ is not too small. Recall that in lowest order $x_{1} x_{2} s=Q^{2}$ so that the parton densities are probed at $\mathrm{x}$ values around $\sqrt{\tau}$. We have $\sqrt{\tau}=0.13-0.15$ (for $\mathrm{W}$ and $\mathrm{Z}$ production, respectively) at $\sqrt{s}=630 \mathrm{GeV}$ (CERN $S p \bar{p} S$ Collider) and $\sqrt{\tau}=0.04-0.05$ at the Tevatron. In this respect the prediction is more delicate at the LHC, where $\sqrt{\tau} \sim 5.7-6.5 \cdot 10^{-3}$. One comparison of the experimental total rates at the Tevatron [48] with the QCD predictions is shown in Fig. 4.19, together with the expected rates at the LHC (based on the structure functions obtained in [23]).

The calculation of the $\mathrm{W} / \mathrm{Z} p_{T}$ distribution has been a classic problem in QCD. For large $p_{T}$, for example $p_{T} \sim o\left(m_{W}\right)$, the $p_{T}$ distribution can be reliably computed in perturbation theory, which was done up to NLO in the late '70's and early '80's. A problem arises in the intermediate range $\Lambda_{Q C D}<<$ $p_{T}<<m_{W}$, where the bulk of the data is concentrated, because terms of order $\alpha_{s}\left(p_{T}^{2}\right) \log m_{W}^{2} / p_{T}^{2}$ become of order one and should included to all orders [39]. At order $\alpha_{s}$ we have:

$$
\frac{1}{\sigma_{0}} \frac{d \sigma_{0}}{d p_{T}^{2}}=(1+A) \delta\left(p_{T}^{2}\right)+\frac{B}{p_{T}^{2}} \log \frac{m_{W}^{2}}{p_{T}^{2}}+\frac{C}{\left(p_{T}^{2}\right)_{+}}+D\left(p_{T}^{2}\right)
$$



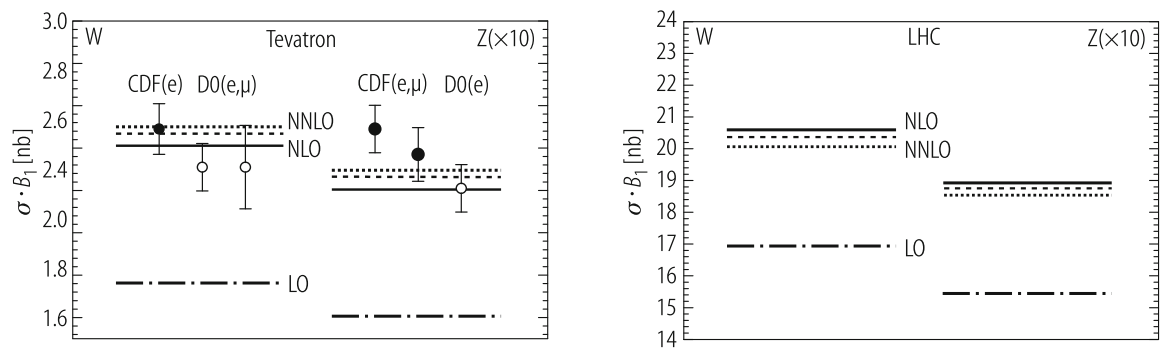

Fig. 4.19 Data vs. theory for $W$ and $Z$ production at the Tevatron $(\sqrt{s}=1.8 \mathrm{TeV})$ together with the corresponding predictions for the LHC $(\sqrt{s}=1.4 \mathrm{TeV})[48]$

where A, B, C, D are coefficients of order $\alpha_{s}$. The " + " distribution is defined in complete analogy with Eq. (4.93):

$$
\int_{0}^{p_{T M A X}^{2}} g(z) f(z)+d z=\int_{0}^{p_{T M A X}^{2}}[g(z)-g(0)] f(z) d z
$$

The content of this, at first sight mysterious, definition is that the singular "+" terms do not contribute to the total cross-section. In fact for the cross-section the weight function $g(z)=1$ and we obtain:

$$
\sigma=\sigma_{0}\left[(1+A)+\int_{0}^{p_{T M A X}^{2}} D(z) d z\right]
$$

The singular terms, of infrared origin, are present at the non completely inclusive level but disappear in the total cross-section. Arguments have been given that these singularities are expected to exponentiate. Explicit calculations in low order support the exponentiation which leads to the following expression:

$$
\frac{1}{\sigma_{0}} \frac{d \sigma_{0}}{d p_{T}^{2}}=\int \frac{d^{2} b}{4 \pi} \exp \left(-i b \cdot p_{T}\right)(1+A) \exp S(b)
$$

with:

$$
S(b)=\int_{0}^{p_{T M A X}} \frac{d^{2} k_{T}}{2 \pi}\left[\exp i k_{T} \cdot b-1\right]\left[\frac{B}{k_{T}^{2}} \log \frac{m_{W}^{2}}{k_{T}^{2}}+\frac{C}{k_{T}^{2}}\right]
$$

At large $p_{T}$ the LO perturbative expansion is recovered. At intermediate $p_{T}$ the infrared $p_{T}$ singularities are resummed (the Sudakov log terms, which are typical of vector gluons, are related to the fact that for a charged particle in acceleration it is impossible not to radiate, so that the amplitude for no soft gluon emission is exponentially suppressed). However this formula has problems at small $p_{T}$, for example, because of the presence of $\alpha_{s}$ under the integral for $S(b)$ : 


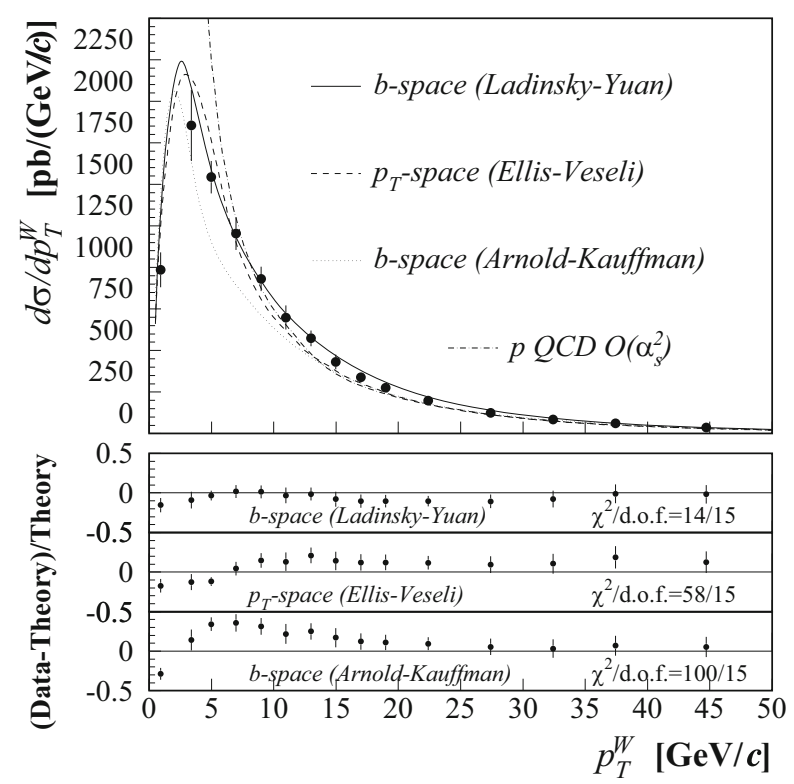

Fig. 4.20 QCD predictions for the $W p_{T}$ distribution compared with recent D0 data at the Tevatron $(\sqrt{s}=1.8 \mathrm{TeV})[49][40]$

presumably the relevant scale is of order $k_{T}^{2}$. So it must be completed by some non perturbative ansatz or an extrapolation into the soft region. All the formalism has been extended to NLO accuracy, where one starts from the perturbative expansion at order $\alpha_{s}^{2}$, and generalises the resummation to also include NLO terms of order $\alpha_{s}\left(p_{T}^{2}\right)^{2} \log m_{W}^{2} / p_{T}^{2}$ (see, for example, [40]). The comparison with the data is very impressive. In Fig. 4.20 we see the $p_{T}$ distribution as predicted in QCD (with a number of variants that mainly differ in the approach to the soft region) compared with some recent data at the Tevatron [49].

A great effort is being devoted to the preparation to the LHC. Calculations for specific processes are being completed. A very important example is Higgs production via $g+g \rightarrow H$. The amplitude is dominated by the top quark loop, as discussed in Chap. 3 [51]. The NLO corrections turn out to be particularly large [52], as seen in Fig. 4.21. Higher order corrections can be computed either in the effective lagrangian approach, where the heavy top is integrated away and the loop is shrunk down to a point [53] [the coefficient of the effective vertex is known to $\alpha_{s}^{4}$ accuracy [54]], or in the full theory. At the NLO the two approaches agree very well for the rate as a function of $m_{H}$ [55]. The NNLO corrections have been computed in the effective vertex approximation [56] (see Fig. 4.21). Beyond fixed order resummation of large logs were carried out [57]. Also the NLO EW contributions have been computed [58]. Rapidity (at NNLO) [59] and $p_{T}$ distributions (at NLO) [60] have also been evaluated. At smaller $p_{T}$ the large $\log \operatorname{arithms}\left[\log \left(p_{T} / m_{H}\right)\right]^{n}$ have been 
Fig. 4.21 The Higgs gluon fusion cross section in LO, NLO and NLLO

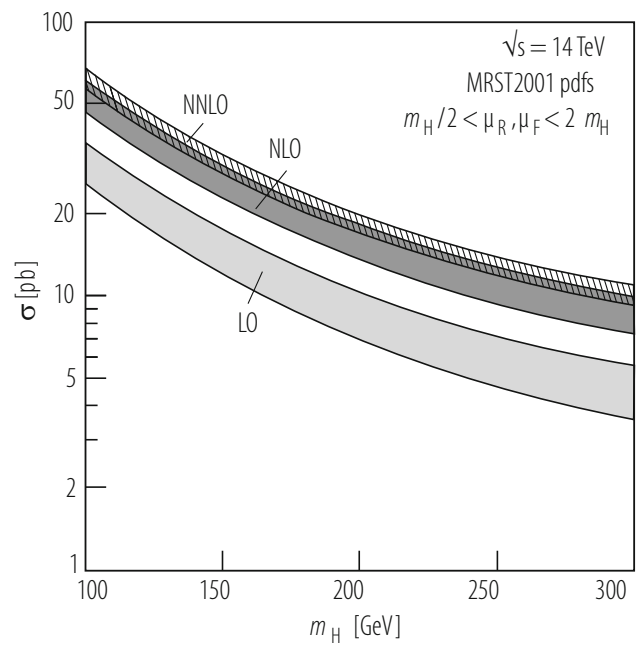

resummed in analogy with what was done long ago for $\mathrm{W}$ and $\mathrm{Z}$ production [61]. For additional recent works on Higgs physics at colliders see, for example, [62].

The activity on event simulation also received a big boost from the LHC preparation (see, for example, the review [50]). General algorithms for performing NLO calculations numerically (requiring techniques for the cancellation of singularities between real and virtual diagrams) have been developed (see, for example, [65]). The matching of matrix element calculation of rates together with the modeling of parton showers has been realised in packages, as for example in the MC@ NLO [63] or POWHEG [64] based on HERWIG. The matrix element calculation, improved by resummation of large logs, provides the hard skeleton (with large $p_{T}$ branchings) while the parton shower is constructed by a sequence of factorized collinear emissions fixed by the QCD splitting functions. In addition, at low scales a model of hadronisation completes the simulation. The importance of all the components, matrix element, parton shower and hadronisation can be appreciated in simulations of hard events compared with the Tevatron data.

At different places in the previous pages we have seen examples of resummation of large logs. This is a very important chapter of modern QCD. The resummation of soft gluon logs enter in different problems and the related theory is subtle. I refer the reader here to some recent papers where additional references can be found [66]. A particularly interesting related development has to do with the so called non global logs (see, for example, [67]). If in the measurement of an observable some experimental cuts are introduced, which is a very frequent case, then a number of large logs can arise from the corresponding breaking of inclusiveness. The discussion of this problem has led to rethinking the theory of final state observables. It is also important to mention the development of software for the automated implementation of resummation (see, for example, [68]). 
Before closing this section I would like to mention some very interesting developments at the interface between string theory and QCD, twistor calculus. A precursor work was the Parke-Taylor result in 1986 [69] on the amplitudes for $n$ incoming gluons with given helicities [70]. Inspired by dual models, they derived a compact formula for the maximum non vanishing helicity violating amplitude (with $\mathrm{n}-2$ plus and 2 minus helicities) in terms of spinor products. Using the relation between strings and gauge theories in twistor space Witten developed in '03 [71] a formalism in terms of effective vertices and propagators that allows to compute all helicity amplitudes. The method, alternative to other modern techniques for the evaluation of Feynman diagrams [73], leads to very compact results. Since then rapid progress followed (for reviews, see [72]): for tree level processes powerful recurrence relations were established [74], the method was extended to include massless external fermions [75] and also external EW vector bosons [76] and Higgs particles [77]. The level already attained is already important for multijet events at the LHC. And the study of loop diagrams has been started. In summary, this road looks new and promising.

\subsection{Measurements of $\alpha_{s}$}

Very precise and reliable measurements of $\alpha_{S}\left(m_{Z}^{2}\right)$ are obtained from $e^{+} e^{-}$colliders (in particular LEP) and from deep inelastic scattering.

\subsection{1 $\alpha_{s}$ from $e^{+} e^{-}$Colliders}

The main methods at $e^{+} e^{-}$colliders are: (a) Inclusive hadronic $\mathrm{Z}$ decay, $R_{l}, \sigma_{h}, \sigma_{l}$, $\Gamma_{Z}$. (b) Inclusive hadronic $\tau$ decay. (c) Event shapes and jet rates.

As we have seen, for a quantity like $R_{l}$ we can write a general expression of the form:

$$
R_{l}=\frac{\Gamma(Z, \tau \rightarrow \text { hadrons })}{\Gamma(Z, \tau \rightarrow \text { leptons })} \sim R^{E W}\left(1+\delta_{Q C D}+\delta_{N P}\right)+\ldots
$$

where $R^{E W}$ is the electroweak-corrected Born approximation, $\delta_{Q C D}, \delta_{N P}$ are the perturbative (logarithmic) and non perturbative (power suppressed) QCD corrections. For a measurement of $\alpha_{s}$ at the $\mathrm{Z}$ (in the following we always refer to the $\overline{M S}$ definition of $\alpha_{S}$ ) one can use all info from $R_{l}, \Gamma_{Z}=3 \Gamma_{l}+\Gamma_{h}$ and ( $\mathrm{f}=\mathrm{h}$ or 1) $\sigma_{f}=12 \pi \Gamma_{l} \Gamma_{f} /\left(m_{Z}^{2} \Gamma_{Z}^{2}\right)$. In the past the measurement from $R_{l}$ was preferred (by itself it leads to $\alpha_{s}\left(m_{Z}\right)=0.1226+0.0058-0.0036$ ) but at LEP there is no reason for that. In all these quantities $\alpha_{s}$ enters through $\Gamma_{h}$, but the measurements of, say, $\Gamma_{Z}, R_{l}$ and $\sigma_{l}$ are really independent (they are affected by entirely different systematics: $\Gamma_{Z}$ is extracted from the line shape, $R_{l}$ and $\sigma_{l}$ are measured at the peak 
but $R_{l}$ does not depend on the absolute luminosity while $\sigma_{l}$ does). The most sensitive single quantity is $\sigma_{l}$. The combined value from the measurements at the $\mathrm{Z}$ (assuming the validity of the SM and a light Higgs mass) is [78]:

$$
\alpha_{s}\left(m_{Z}\right)=0.119 \pm 0.003
$$

For a relatively light Higgs (even if not as light as from the fit to EW observables) the final error is mainly experimental with a theoretical component from our ignorance of $m_{H}$, of higher orders in the QCD expansion [79] and also from uncertainties on the Bhabha luminometer (which affect $\sigma_{h, l}$ ) [80]. By adding all other electroweak precision electroweak tests (in particular $m_{W}$ ) one similarly finds [41]:

$$
\alpha_{S}\left(m_{Z}\right)=0.1185 \pm 0.003
$$

We now consider the measurement of $\alpha_{s}\left(m_{Z}\right)$ from $\tau$ decay. $R_{\tau}$ has a number of advantages that, at least in part, tend to compensate for the smallness of $m_{\tau}=$ $1.777 \mathrm{GeV}$. First, $R_{\tau}$ is maximally inclusive, more than $R_{e^{+} e^{-}}(s)$, because one also integrates over all values of the invariant hadronic squared mass:

$$
R_{\tau}=\frac{1}{\pi} \int_{0}^{m_{\tau}^{2}} \frac{d s}{m_{\tau}^{2}}\left(1-\frac{s}{m_{\tau}^{2}}\right)^{2} \operatorname{Im} \Pi_{\tau}(s)
$$

The perturbative contribution is known at NNLO. Analyticity can be used to transform the integral into one on the circle at $|s|=m_{\tau}^{2}$ :

$$
R_{\tau}=\frac{1}{2 \pi i} \oint_{|s|=m_{\tau}^{2}} \frac{d s}{m_{\tau}^{2}}\left(1-\frac{s}{m_{\tau}^{2}}\right)^{2} \Pi_{\tau}(s)
$$

Also, the factor $\left(1-\frac{s}{m_{\tau}^{2}}\right)^{2}$ is important to kill the sensitivity the region $\operatorname{Re}[s]=m_{\tau}^{2}$ where the physical cut and the associated thresholds are located. Still the quoted result [81] looks a bit too precise:

$$
\alpha_{S}\left(m_{\tau}\right)=0.345 \pm 0.010
$$

or

$$
\alpha_{S}\left(m_{Z}\right)=0.1215 \pm 0.0012
$$

This precision is obtained by taking for granted that corrections suppressed by $1 / m_{\tau}^{2}$ are negligible. This is because, in the massless theory, the light cone expansion is given by:

$$
\delta_{N P}=\frac{Z E R O}{m_{\tau}^{2}}+c_{4} \cdot \frac{<O_{4}>}{m_{\tau}^{4}}+c_{6} \cdot \frac{<O_{6}>}{m_{\tau}^{6}}+\cdots
$$


In fact there are no dim-2 Lorentz and gauge invariant operators. For example, $g_{\mu} g^{\mu}$ is not gauge invariant. In the massive theory, the ZERO is replaced by light quark mass-squared $m^{2}$. This is still negligible if $m$ is taken as a lagrangian mass of a few MeV. If on the other hand the mass were taken to be the constituent mass of order $\Lambda_{Q C D}$, this term would not be at all negligible and would substantially affect the result (note that $\alpha_{S}\left(m_{\tau}\right) / \pi \sim 0.1 \sim\left(0.6 \mathrm{GeV} / m_{\tau}\right)^{2}$ and that $\Lambda_{Q C D}$ for three flavours is large). For example, the PDG value and estimate of the error is [9]:

$$
\alpha_{S}\left(m_{Z}\right)=0.120 \pm 0.003 \text {. }
$$

Most people believe the optimistic version. I am not convinced that the gap is not filled up by ambiguities of $0\left(\Lambda_{Q C D}^{2} / m_{\tau}^{2}\right)$ from $\delta_{\text {pert }}$ [82]. In any case, one can discuss the error, but it is true and remarkable, that the central value from $\tau$ decay, obtained at very small $Q^{2}$, is in good agreement with all other precise determinations of $\alpha_{s}$ at more typical LEP values of $Q^{2}$.

Important determinations of $\alpha_{S}\left(m_{Z}\right)$ are obtained from different infrared safe observables related to event rates and jet shapes in $e^{+} e^{-}$annihilation. The main problem of these measurements is the large impact of non perturbative hadronization effects on the result and therefore on the theoretical error. The perturbative part is known at NLO. One advantage is that the same measurements can be repeated at different $\sqrt{s}$ values (e.g. with the same detectors at LEP1 or LEP2) allowing for a direct observation of the energy dependence. A typical result, from jets and event shapes at LEP, quoted in Ref. [83], is given by:

$$
\alpha_{S}\left(m_{Z}\right)=0.121 \pm 0.005 \text {. }
$$

Recently the rate of 4-jet events (proportional to $\alpha_{s}^{2}$ ) at LEP as function of $y_{\text {cut }}$ has been used [84], for which a NLO theoretical calculation exists [85]. The quoted result is $\alpha_{S}\left(m_{Z}\right)=0.1176 \pm 0.0022$ (the actual error could be somewhat larger because the ambiguity from hadronisation modeling is always debatable).

\subsection{2 $\alpha_{s}$ from Deep Inelastic Scattering}

QCD predicts the $Q^{2}$ dependence of $F\left(x, Q^{2}\right)$ at each fixed x, not the x shape. But the $Q^{2}$ dependence is related to the x shape by the QCD evolution equations. For each x-bin the data allow to extract the slope of an approximately straight line in $d \log F\left(x, Q^{2}\right) / d \log Q^{2}$ : the $\log$ slope. The $Q^{2}$ span and the precision of the data are not much sensitive to the curvature, for most $\mathrm{x}$ values. A single value of $\Lambda_{Q C D}$ must be fitted to reproduce the collection of the log slopes. For the determination of $\alpha_{s}$ the scaling violations of non-singlet structure functions would be ideal, because 
of the minimal impact of the choice of input parton densities. We can write the non-singlet evolution equations in the form:

$$
\frac{d}{d t} \log F(x, t)=\frac{\alpha_{s}(t)}{2 \pi} \int_{x}^{1} \frac{d y}{y} \frac{F(y, t)}{F(x, t)} P_{q q}\left(\frac{x}{y}, \alpha_{s}(t)\right)
$$

where $P_{q q}$ is the splitting function. At present NLO and NNLO corrections are known. It is clear from this form that, for example, the normalisation error on the input density drops away, and the dependence on the input is reduced to a minimum (indeed, only a single density appears here, while in general there are quark and gluon densities). Unfortunately the data on non-singlet structure functions are not very accurate. If we take the difference of data on protons and neutrons, $F_{p}-F_{n}$, experimental errors add up in the difference and finally are large. The $F_{3 v N}$ data are directly non-singlet but are not very precise. A determination of $\alpha_{s}$ from the CCFR data on $F_{3 v N}$ has led to [86]:

$$
\alpha_{s}\left(m_{Z}\right)=0.119 \pm 0.006
$$

A recent analysis of the same data leads to $\alpha_{S}\left(m_{Z}\right)=0.119 \pm 0.002$ [87], but the theoretical error associated with the method and with the choice adopted for the scale ambiguities is not considered. A fit to non singlet structure functions in electroor muon-production extracted from proton and deuterium data at the NNLO level was performed in Ref. [88] with the result:

$$
\alpha_{S}\left(m_{Z}\right)=0.114 \pm 0.002
$$

When one measures $\alpha_{s}$ from scaling violations on $F_{2}$ from e or $\mu$ beams, the data are abundant, the errors small but there is an increased dependence on input parton densities and especially a strong correlation between the result on $\alpha_{s}$ and the input on the gluon density. There are complete and accurate derivations of $\alpha_{s}$ from scaling violations in $F_{2}$. In a well known analysis by Santiago and Yndurain [89], the data on protons from SLAC, BCDMS, E665 and HERA are used with NLO kernels plus the NNLO first few moments. The analysis is based on an original method that uses projections on a specially selected orthogonal basis, the Bernstein polynomials. The quoted result is given by:

$$
\alpha_{S}\left(m_{Z}\right)=0.1163 \pm 0.0014
$$

(these authors also quote $\alpha_{s}\left(m_{Z}\right)=0.115 \pm 0.006$ for $F_{3}$ data in $v N$ scattering). A different analysis by Alekhin [90] of existing data off proton and deuterium targets with NNLO kernels and a more conventional method leads to

$$
\alpha_{s}\left(m_{Z}\right)=0.114 \pm 0.002
$$


In both analyses the dominant error is theoretical and, in my opinion, should be somewhat larger than quoted. An interesting perspective on theoretical errors can be obtained by comparing analyses with different methods. We add the following examples. From truncated moments (but with a limited set of proton data and NLO kernels) [91]: $\alpha_{S}\left(m_{Z}\right)=0.122 \pm 0.006$, from Nachtmann moments (which take into account some higher twist terms) and proton data [92]: $\alpha_{s}\left(m_{Z}\right)=0.1188 \pm 0.0017$. A combination of measurements at HERA by $\mathrm{H} 1$ and Zeus, also including final state jet observables, leads to $\alpha_{s}\left(m_{Z}\right)=0.1186 \pm 0.0051$ [93], most of the error being theoretical. Finally, to quote a number that appears to me as a good summary of the situation of $\alpha_{s}\left(m_{Z}\right)$ from DIS one can take the result from a NNLO analysis of available data by the MRST group [94] as quoted by Particle Data Group, W.-M. Yao et al. [9]:

$$
\alpha_{S}\left(m_{Z}\right)=0.1167 \pm 0.0036
$$

If we compare these results on $\alpha_{s}$ from DIS with the findings at the Z, given by Eq. (4.105), we see that the agreement is good, with the value of $\alpha_{s}$ from the most precise DIS measurements a bit on the low side with respect to $e^{+} e^{-}$.

\subsubsection{Summary on $\alpha_{s}$}

There are a number of other determinations of $\alpha_{s}$ which are important because they arise from qualitatively different observables and methods. For example [9, 83], some are obtained from the Bjorken sum rule and the scaling violations in polarized DIS, from $\Upsilon$ decays, from the 4-jet rate in $e^{+} e^{-}$. A special mention deserves the "measurement" of $\alpha_{s}$ from lattice QCD [95]. A number of hadronic observables, in particular $\Upsilon^{\prime}-\Upsilon$ splitting, pion and kaon decay constants, the $B_{S}$ mass and the $\Omega$ baryon mass are used to fix the lattice spacing and to accurately tune the QCD simulation. The value of $\alpha_{s}$ is then obtained by computing non perturbatively a number of quantities related to Wilson loops that can also be given in perturbation theory. The result is then evolved with state of the art beta functions to obtain $\alpha_{s}\left(m_{Z}\right)=0.1170 \pm 0.0012$. This result is interesting for its really special nature but it is not clear that the systematics due to the lattice technology is as small as claimed.

Summarising: there is very good agreement among many different measurements of $\alpha_{s}$. In Fig. 4.22 [83], a compilation of the data is reported with each measurement plotted at the scale of the experiment, which shows the consistency of the measurements and the running of $\alpha_{s}$. This is a very convincing, quantitative test of QCD. If I take the values of $\alpha_{S}\left(m_{Z}\right)$ from precision electroweak data, Eq. (4.105), from $\tau$ decay with the central value as in Eq. (4.109) but the larger error as in Eq. (4.111), from jets in $e^{+} e^{-}$, Eq. (4.112), and from DIS, Eq. (4.118), the average is :

$$
\alpha_{s}\left(m_{Z}\right)=0.119 \pm 0.002
$$


Fig. 4.22 The running of $\alpha_{s}$ as determined from present data [83]

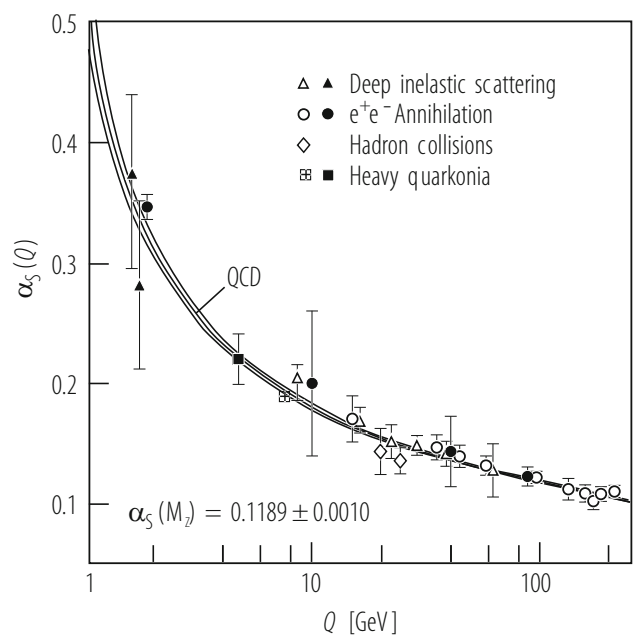

For comparison, the average value quoted by PDG 2006 is $\alpha_{S}\left(m_{Z}\right)=0.1176 \pm$ 0.0020 while Ref. [83] gives $\alpha_{s}\left(m_{Z}\right)=0.1189 \pm 0.0010$.

The value of $\Lambda$ (for $n_{f}=5$ ) which corresponds to Eq. (4.119) is:

$$
\Lambda_{5}=221 \pm 25 \mathrm{MeV}
$$

$\Lambda$ is the scale of mass that finally appears in massless QCD. It is the scale where $\alpha_{s}(\Lambda)$ is of order one. Hadron masses are determined by $\Lambda$. Actually the $\rho$ mass or the nucleon mass receive little contribution from the quark masses (the case of pseudoscalar mesons is special, as they are the pseudo Goldstone bosons of broken chiral invariance). Hadron masses would be almost the same in massless QCD.

\subsection{Conclusion}

We have seen that perturbative QCD based on asymptotic freedom offers a rich variety of tests and we have described some examples in detail. QCD tests are not as precise as for the electroweak sector. But the number and diversity of such tests has established a very firm experimental foundation for QCD as a theory of strong interactions. The field of QCD appears as one of great maturity but also of robust vitality with many rich branches and plenty of new blossoms. The physics content of QCD is very large and our knowledge, especially in the non perturbative domain, is still very limited but progress both from experiment (Tevatron, RHIC, LHC......) and from theory is continuing at a healthy rate. And all the QCD predictions that we were able to formulate and to test appear to be in very good agreement with experiment. 
Acknowledgments I am very grateful to Richard Ball, Keith Ellis, Stefano Forte, Ashutosh Kotwal, Vittorio Lubicz, Lorenzo Magnea, Michelangelo Mangano, Silvano Simula, Hartmut Wittig for their help and advise.

\section{References for 4}

Disclaimer: This list of references is by far incomplete. It is just meant to provide a guide to the vast literature on QCD. So it includes only a few old papers and, for most of the issues, some relatively recent works where to look for a more complete list of references.

\section{References}

1. M. Gell-Mann, Acta Phys. Austriaca Suppl. IX (1972) 733; H. Fritzsch and M. Gell-Mann, Proc. XVI Int. Conf. on High Energy Physics, Chicago-Batavia, 1972; H. Fritzsch, M. GellMann, H. Leutwyler, Phys. Lett. B47 (1973) 365.

2. D.J. Gross, F. Wilczek, Phys. Rev. Lett. 30 (1973) 1343; Phys. Rev. D8 (1973) 3633; H.D. Politzer, Phys. Rev. Lett. 30 (1973) 1346.

3. S. Weinberg, Phys. Rev. Lett. 31 (1973) 494.

4. G. Altarelli, Phys. Rep. 81(1982)1.

5. G. Altarelli, Ann. Rev. Nucl. Part. Sci. 39(1989)357.

6. G. Altarelli, The Development of Perturbative QCD, World Scientific, (1994); W. Greiner, S. Schramm and E. Stein, Quantum Chromodynamics, Springer, 1994; Handbuch of QCD. ed. by M. Shifman, Vol.1-4, World Sci. (2001); R. K. Ellis, W. J. Stirling, and B. R. Webber, $Q C D$ and Collider Physics, Cambridge Monographs, (2003); G. Dissertori, I. Knowles and M. Schmelling, Quantum Chromodynamics: High Energy Experiments and Theory, Oxford Univ. Press, 2003.

7. M. Gell-Mann, Phys. Lett. 8 (1964) 214 ; G. Zweig, CERN TH 401 and 412 (1964). O.W. Greenberg,Phys. Rev. Lett. 13 (1964) 598.

8. F. Bloch and H. Nordsieck, Phys. Rev. 52(1937)54.

9. Particle Data Group, W.-M. Yao et al., Journal of Physics G 33, 1 (2006).

10. T. Kinoshita, J. Math. Phys. 3(1962)650; T.D. Lee and M. Nauenberg, Phys. Rev. 133(1964)1549.

11. T. Applequist and J. Carazzone, Phys. Rev. D11 (1975)2856.

12. O.V. Tarasov, A.A. Vladimirov and A. Yu. Zharkov, Phys. Lett. 93B(1980)429.

13. T. van Ritbergen, J.A.M. Vermaseren and S.A. Larin, Phys.Lett.B400(1997)379; see also M. Czakon, Nucl. Phys. B710 (2005) 485.

14. G. Altarelli, Proceedings of the E. Majorana Summer School, Erice, 1995, Plenum Press, ed. by A. Zichichi; M. Beneke and V. M. Braun, in Handbuch of QCD. ed. by M. Shifman, Vol.3, pag. 1719, World Sci. (2001).

15. S.G. Gorishny, A.L. Kataev and S.A. Larin, Phys. Lett. 259B (1991) 144; L. R. Surguladze and M. A. Samuel, Phys. Rev. Lett. 66 (1991) 560.

16. R. Brandt and G. Preparata,Nucl. Phys. 27B (1971) 541.

17. Yu. Dokshitzer, J. Phys.G17 (1991)1572; N. Brown and W. J. Stirling, Z. Phys. C53 (1992) 629.

18. V.N. Gribov and L.N. Lipatov, Sov. J. Nucl. Phys. 15 (1972) 438; see also Yu. L. Dokshitzer, Sov. Phys. JETP 46 (1977) 641.

19. G.Altarelli and G. Parisi, Nucl. Phys. B126 (1977) 298.

20. E. G. Floratos, D.A. Ross and C. T. Sachrajda, Nucl. Phys. B129 (1977) 66, E B139 (1978) 545; B152 (1979) 493; A. Gonzales-Arroyo, C. Lopez and F. J. Yndurain, Nucl. Phys. B153 (1979) 161; G. Curci, W. Furmanski and R. Petronzio, Nucl. Phys. B175 (1980) 27; W. Furmanski 
and R. Petronzio, Phys. Lett. 97B (1980) 438; E.G. Floratos, R. Lacaze and C. Kounnas, Phys. Lett. 99B (1981) 89, 285; R. T. Herrod and S. Wada, Z. Phys. C9 (1981) 351.

21. S. Moch, J. A. M. Vermaseren and A. Vogt, Nucl. Phys. B 688, 101 (2004), [arXiv:hepph/0403192]; A. Vogt, S. Moch and J. A. M. Vermaseren, Nucl. Phys. B 691, 129 (2004), [arXiv:hep-ph/0404111].

22. 5) G. Altarelli, R.Keith Ellis, G. Martinelli, Nucl.Phys.B143:521,1978, E.ibid.B146:544,1978; Nucl.Phys.B157:461,1979.

23. A. D. Martin, R. G. Roberts, W. J. Stirling and R. S. Thorne, hep-ph/0110215, hep-ph/0201127.

24. G. Sterman, Nucl. Phys. B281 (1987) 310; S. Catani and L. Trentadue, Nucl. Phys. B327 (1989) 323; N. Kidonakis and G. Sterman, Nucl. Phys. B505 (1997) 321.

25. S. Forte and G. Ridolfi, Nucl.Phys. B650:229,2003; S. Moch, J. A. M. Vermaseren and A. Vogt, Nucl. Phys. B 726 (2005) 317 [arXiv:hep-ph/0506288]; T. Becher, M. Neubert and B. D. Pecjak, JHEP 0701 (2007) 076 [arXiv:hep-ph/0607228].

26. E. Gardi, G. P. Korchemsky, D. A. Ross and S. Tafat, Nucl. Phys. B 636 (2002) 385 [arXiv:hepph/0203161]; E. Gardi and R. G. Roberts, Nucl. Phys. B 653 (2003) 227 [arXiv:hep$\mathrm{ph} / 0210429]$.

27. L.N. Lipatov, Sov. Jour. Nucl. Phys. 23 (1976) 338; V.S. Fadin, E.A. Kuraev and L.N. Lipatov, Phys. Lett. 60B (1975) 50; Sov. Phys. JETP 44 (1976) 443; 45(1977)199; Y.Y. Balitski and L.N.Lipatov, Sov. Jour. Nucl. Phys. 28 (1978) 822.

28. V.S. Fadin and L.N. Lipatov, Phys. Lett. B429 (1998) 127; B429 (1998) 127; V.S. Fadin et al, Phys. Lett. B359 (1995)181; B387 (1996)593; Nucl. Phys. B406 (1993) 259; Phys. Rev. D50 (1994) 5893; Phys. Lett. B389 (1996) 737; Nucl. Phys. B477 (1996) 767; Phys. Lett. B415 (1997) 97; B422 (1998) 287; G. Camici and M. Ciafaloni, Phys. Lett. B412 (1997) 396; Phys. Lett. B430 (1998) 349. V. del Duca, Phys. Rev. D54 (1996) 989; D54 (1996) 4474; V. del Duca and C.R. Schmidt, Phys. Rev. D57 (1998) 4069; Z. Bern, V. del Duca and C.R. Schmidt, Phys. Lett. B445 (1998) 168.

29. G. Altarelli, R. Ball and S. Forte, Nucl. Phys. B742 (2006) 1.

30. M. Ciafaloni, D. Colferai, G. P. Salam and A. M. Stasto, Phys. Lett. B587 (2004) 87; see also G. P. Salam, hep-ph/0501097; C. D. White and R. S. Thorne, Phys. Rev. D 75 (2007) 034005 [hep-ph/0611204].

31. S. Forte and Y. Goto, Proceedings of DIS 2006, Tsukuba, Japan, World Scientific, (2007), p.908 [hep-ph/0609127].

32. M. Hirai, S. Kumano and N. Saito, Phys.Rev. D69 (2004) 05402.

33. G. Mallot, hep-ex/0612055.

34. G.Altarelli and G. G. Ross, Phys. Lett. B212 (1988) 391; A. V. Efremov and O. V. Terayev, Proceedings of the Czech Hadron Symposium, 1988, p. 302; R. D. Carlitz, J. C. Collins and A. H. Mueller, Phys. Lett. B214 (1988) 229; G. Altarelli and B. Lampe, Z. Phys. C47 (1990) 315.

35. CDF Run II Collaboration, Phys.Rev.D74:071103,2006; e-Print: hep-ex/0512020 the D0 Collaboration, Phys.Rev.Lett.86:2523,2001; Phys.Lett.B639:151,2006.

36. The CDF Collaboration, Phys.Rev.Lett.73:2662-2666,1994, Erratum-ibid.74:18911893,1995; Phys.Rev.D65:112003,2002; Phys.Rev.D70:074008,2004;

37. R. Ha the D0 Collaboration, Phys.Rev.Lett.87:251805,2001; Phys.Rev.Lett.84:2786,2000.

38. J. Kubar-Andre' and F. Paige, Phys. Rev. D19(1979) 221. mberg, W. L. van Neerven and T. Matsuura, Nucl. Phys. B359 (1991) 343; W. L. van Neerven and E. B. Zijlstra, Nucl. Phys. B382(1992) 11.

39. V.V. Sudakov, Sov. Phys. JETP, 3 (1956) 75; Yu. Dokshitzer, D. Dyakonov and S. Troyan, Phys. Lett. 76B (1978) 290, Phys. Rep. 58 (1980 269; G. Parisi and R. Petronzio, Nucl. Phys. B154 ((1979) 427; G. Curci, M. Greco and Y. Srivastava, Phys. Rev. Lett. 43 (1979), Nucl. Phys. B159 (1979) 451; J. Collins and D. Soper, Nucl. Phys. B139 (1981) 381, B194 (1982) 445 , B197 (1982)446; J. Kodaira and L. Trentadue, Phys. Lett. 112B (1982) 66, 123B (1983)335; J. Collins, D. Soper and G. Sterman, Nucl. Phys. B250 (1985) 199; C. Davies, B. Webber and J. Stirling, Nucl. Phys. B256 (1985) 413.

40. P. Arnold and R. Kauffman, Nucl.Phys.B349:381,1991; G.A. Ladinsky and C.P. Yuan, Phys.Rev.D50:4239,1994; R.K. Ellis, S. Veseli, Nucl.Phys.B511:649,1998. 
41. For up-to-date results see, The LEP Electroweak Group, http://lepewwg.web.cern.ch/ LEPEWWG/.

42. P. Nason, S. Dawson and R. K. Ellis, Nucl. Phys. B 303 (1988) 607; W. Beenakker, H. Kuijf, W. L. van Neerven and J. Smith, Phys. Rev. D 40 (1989) 54; P. Nason, S. Dawson and R. K. Ellis, Nucl. Phys. B 327 (1989) 49 [Erratum-ibid. B 335 (1989) 260]; M. L. Mangano, P. Nason and G. Ridolfi, Nucl. Phys. B 373 (1992) 295; R. Bonciani, S. Catani, M. L. Mangano and P. Nason, Nucl.Phys. B529:424,1998; M. Cacciari et al, JHEP 0404:068, 2004; N. Kidonakis and A. Vogt, Phys. Rev. D68 114014(2003); A. Banfi, G. P. Salam and G. Zanderighi, JHEP 0707 (2007) 026 [arXiv:0704.2999 [hep-ph]].

43. The CDF II Collaboration, Phys.Rev.D74:072005,2006; Phys.Rev.D74:072006,2006; Phys.Rev.D71:072005,2005; the CDF Collaboration, Phys.Rev.Lett.97:082004,2006; Phys.Rev.Lett.96:202002,2006; Phys.Rev.D72:052003,2005; Phys.Rev.D71:052003,2005; Phys.Rev.Lett.93:142001,2004. The D0 Collaboration, Phys.Rev.D74:112004,2006; Phys.Lett.B626:55,2005; Phys.Lett.B626:35,2005; Phys.Lett.B626:45,2005.

44. The CDF Collaboration, CDF Note 8148, v. 1.0, 2006.

45. The CDF Collaboration, Phys.Rev.D75:012010,2007; Phys.Rev.D71:032001,2005; Phys.Rev.D65:052005,2002; the D0 Collaboration, Phys.Rev.Lett.94:232001,2005; Phys.Rev.Lett.85:5068,2000; Phys.Lett.B487:264-272,2000.

46. M. Cacciari et al, JHEP 0407:033, 2004.

47. D. Bauer (for the CDF and D0 Collaborations), Nucl. Phys. B (Proc. Suppl.) 156 (2006) 226.

48. The CDF II Collaboration, Phys.Rev.Lett. 94:091803, 2005; the D0 Collaboration, Phys.Rev. D60:052003,1999.

49. The D0 collaboration, Phys. Rev. Lett. 84 (2000) 2792; Phys.Rev. D61 (2000) 032004; see also the CDF Collaboration, Phys.Rev.Lett. 84 (2000) 845.

50. J.M. Campbell, J.W. Huston and W.J. Stirling, Rept.Prog.Phys. 70 (2007) 89

51. H.Georgi, S.Glashow, M.Machacek and D.Nanopoulos,Phys.Rev.Lett. 40,692(1978)

52. S. Dawson, Nucl. Phys. B 359, 283 (1991); A. Djouadi, M. Spira and P. M. Zerwas, Phys. Lett. B 264, 440 (1991); M. Spira, A. Djouadi, D. Graudenz and P. Zerwas, Nucl. Phys. B 453, 17 (1995).

53. J. Ellis, M. Gaillard and D. Nanopoulos, Nucl. Phys. B 106, 292 (1976)

54. K. Chetyrkin, B. Kniehl and M. Steinhauser, Phys. Rev. Lett. 79, 353 (1997)

55. M. Kramer, E. Laenen and M. Spira, Nucl. Phys. B 511, 523 (1998)

56. S.Catani, D.de Florian and M.Grazzini, JHEP0105,025(2001); JHEP0201,015(2002); R. V. Harlander, Phys. Lett. B 492, 74 (2000); V. Ravindran, J. Smith and W. L. van Neerven, Nucl. Phys. B 704, 332 (2005); R. V. Harlander and W. B. Kilgore, Phys. Rev. D 64, 013015 (2001); R. V. Harlander and W. B. Kilgore, Phys. Rev. Lett. 88, 201801 (2002); C. Anastasiou and K. Melnikov, Nucl. Phys. B 646, 220 (2002); V. Ravindran, J. Smith and W. L. van Neerven, Nucl. Phys. B 665, 325 (2003).

57. S. Catani, D. de Florian, M. Grazzini and P. Nason, JHEP 0307, 028 (2003); S. Moch and A. Vogt, Phys. Lett. B 631, 48 (2005); V. Ravindran, Nucl. Phys. B 752, 173 (2006) (and references therein).

58. U. Aglietti, R. Bonciani, G. Degrassi and A. Vicini, Phys. Lett. B 595, 432 (2004); G. Degrassi and F. Maltoni, Phys. Lett. B 600, 255 (2004).

59. C. Anastasiou, K. Melnikov and F. Petriello, Nucl. Phys. B 724, 197 (2005); V. Ravindran, J. Smith and W. L. van Neerven, Nucl. Phys. B 767 (2007) 100 [hep-ph/0608308].

60. D. de Florian, M. Grazzini and Z. Kunszt, Phys. Rev. Lett. 82, 5209 (1999); V. Ravindran, J. Smith and W. L. Van Neerven, Nucl. Phys. B 634, 247 (2002); C. J. Glosser and C. R. Schmidt, JHEP 0212, 016 (2002).

61. G. Bozzi, S. Catani, D. de Florian and M. Grazzini, Phys. Lett. B 564, 65 (2003); Nucl. Phys. B 737, 73 (2006); A. Kulesza, G. Sterman and W. Vogelsang, Phys. Rev. D 69, 014012 (2004).

62. R.K. Ellis, W.T. Giele and G. Zanderighi, Phys.Rev. D72 (2005) 054018; E. D74 (2006) 079902; J. M. Campbell, R. K. Ellis and G. Zanderighi, JHEP 0610 (2006) 028; C. Anastasiou et al, JHEP 0701 (2007) 082; G. Davatz et al, JHEP 0607 (2006) 037; S. Catani and M. Grazzini, Phys. Rev. Lett. 98 (2007) 222002; C. Anastasiou, G. Dissertori and F. Stoeckli, 
JHEP 0709 (2007) 018 [arXiv:0707.2373 [hep-ph]]; P. M. Nadolsky, C. Balazs, E. L. Berger and C.-P. Yuan, Phys. Rev. D 76 (2007) 013008 [hep-ph/0702003 [HEP-PH]]; C. Balazs, E. L. Berger, P. M. Nadolsky and C.-P. Yuan, Phys. Rev. D 76 (2007) 013009 [arXiv:0704.0001 [hep-ph]]; M. Ciccolini, A. Denner and S. Dittmaier, Phys. Rev. Lett. 99 (2007) 161803 [arXiv:0707.0381 [hep-ph]].

63. S. Frixione and B. Webber, hep-ph/0612272 and references therein.

64. S. Frixione, P. Nason and G. Ridolfi, arXiv:0707.3081; S. Frixione, P. Nason and G. Ridolfi, JHEP 0709 (2007) 126 [arXiv:0707.3088 [hep-ph]]

65. K. Fabricius, I. Schmitt, G. Kramer and G. Schierholz, Z. Phys. C 11 (1981) 315; G. Kramer and B. Lampe, Fortsch. Phys. 37 (1989) 161; R. K. Ellis, D. A. Ross and A. E. Terrano, Nucl. Phys. B 178 (1981) 421; S. Frixione, Z. Kunszt and A. Signer, Nucl.Phys.B467:399,1996; D. Kosower, Phys.Rev.D71:045016,2005; S. Catani, S. Dittmaier, M. H. Seymour and Z. Trócsányi, Nucl.Phys.B627,189 (2002).

66. A. Banfi and M. Dasgupta, JHEP 0401 (2004) 027; P Bolzoni, S. Forte and G. Ridolfi, Nucl.Phys.B731:85,2005; Y. Delenda, R. Appleby, M. Dasgupta and A. Banfi, JHEP 0612 (2006) 044; P. Bolzoni, Phys.Lett.B643:325,2006; S. Mert Aybat, L. J. Dixon and G. Sterman, Phys. Rev. D 74 (2006) 074004; Yu. L. Dokshitzer and G. Marchesini, JHEP 0601 (2006) 007; E. Laenen and L. Magnea, Phys. Lett. B 632 (2006) 270; C. Lee and G. Sterman, Phys. Rev. D 75, 014022 (2007); D. de Florian and W. Vogelsang, Phys. Rev. D 76 (2007) 074031 [arXiv:0704.1677 [hep-ph]]; R. Abbate, S. Forte and G. Ridolfi, Phys. Lett. B 657 (2007) 55 [arXiv:0707.2452 [hep-ph]].

67. M. Dasgupta and G. P. Salam, Phys. Lett. B 512 (2001) 323; M. Dasgupta and G. P. Salam, J. Phys. G 30 (2004) R143; A. Banfi, G. Corcella, M. Dasgupta, Y. Delenda, G. P. Salam and G. Zanderighi, hep-ph/0508096; J. R. Forshaw, A. Kyrieleis and M. H. Seymour, JHEP 0608 (2006) 059.

68. A. Banfi, G. P. Salam and G. Zanderighi, JHEP 0503 (2005) 073.

69. S.J. Parke and T.R. Taylor, Phys.Rev.Lett.56:2459,1986.

70. F.A. Berends and W. Giele, Nucl. Phys. B294 (1987) 700; M. L. Mangano, S.J. Parke and Z. Xu, Nucl. Phys. B298 (1988) 653.

71. E. Witten, Commun.Math.Phys.252:189,2004, [hep-th/0312171].

72. F. Cachazo and P. Svrcek, PoS RTN2005:004,2005, [hep-th/0504194]; L. J. Dixon, Proceedings of the EPS International Europhysics Conference on High Energy Physics, Lisbon, Portugal, 2005, PoS HEP2005:405,2006, [hep-ph/0512111] and references therein.

73. M. Dinsdale, M. Ternick and S. Weinzierl, JHEP 0603:056,2006, [hep-ph/0602204].

74. R. Roiban, M. Spradlin and A. Volovich, Phys. Rev. Lett. 94102002 (2005); R. Britto, F. Cachazo and B. Feng, Nucl. Phys. B715 (2005) 499; R. Britto, F. Cachazo, B. Feng and E. Witten, Phys. Rev. Lett. 94181602 (2005).

75. G. Georgiou and V. V. Khoze, JHEP 0405 (2004) 015; J. B. Wu and C.J. Zhu, JHEP 0409 (2004) 063; G. Georgiou, E. W. N. Glover and V. V. Khoze, JHEP 0407 (2004) 048.

76. Z. Bern, D. Forde, D. A. Kosower and P. Mastrolia, Phys.Rev.D72:025006,2005.

77. L. J. Dixon, E. W. N. Glover and V. V. Khoze, JHEP 0412 (2004) 015; S. D. Badger, E. W. N. Glover and V. V. Khoze, JHEP 0503 (2005) 023; S.D. Badger, E.W.N. Glover and K. Risager, Acta Phys.Polon.B38:2273-2278,2007.

78. S. Kluth, Rept. Prog. Phys. 69 (2006) 1771.

79. H. Stenzel, JHEP 0507:0132,2005.

80. W. de Boer and C. Sander, Phys.Lett.B585:276,2004.

81. M. Davier, A. Hocker and Z. Zhang, Rev.Mod.Phys.78:1043,2006, [hep-ph/0507078].

82. G Altarelli, P. Nason and G. Ridolfi, Z.Phys.C68:257,1995.

83. S. Bethke, Prog.Part.Nucl.Phys.58:351,2007, [hep-ex/0606035].

84. ALEPH Collaboration, A. Heister et al, Eur. J. Phys. C 27 (2003) 1; DELPHI Collaboration, J. Abdallah et al, Eur. J. Phys. C 38 (2005) 413; OPAL Collaboration, G. Abbiendi et al, hepex/0601048.

85. Z. Nagy and Z. Trocsanyi, Phys. Rev. D59 (1999) 14020, E. D62 (2000) 099902. 
86. A.L. Kataev, G. Parente and A.V. Sidorov, Nucl.Phys. B573 (2000) 405; Nucl.Phys.Proc.Suppl.116:105,2003,[hep-ph/0211151].

87. P. M. Brooks and C.J. Maxwell, Nucl.Phys.B780:76, 2007, [hep-ph/0610137].

88. J. Blumlein, H. Bottcher and A. Guffanti, Nucl.Phys.B774:182,2007, [hep-ph/0607200].

89. J. Santiago and F.J. Yndurain, Nucl.Phys. B563 (1999) 45; Nucl. Phys. Proc. Suppl. 86 (2000)69; Nucl.Phys. B611 (2001) 45.

90. S.I. Alekhin, Phys.Rev. D59:114016,1999.

91. S. Forte, J. I. Latorre, L. Magnea and A. Piccione, Nucl.Phys.B643:477,2002, [hep$\mathrm{ph} / 0205286]$.

92. S. Simula, M. Osipenko, Nucl.Phys.B675:289,2003, [hep-ph/0306260].

93. C. Glasman, hep-ex/0506035.

94. A.D. Martin, R.G. Roberts, W.J. Stirling and R.S. Thorne, Eur.Phys.J.C35:325, 2004, [hep$\mathrm{ph} / 0308087]$.

95. Q. Mason et al, Phys. Rev. Lett. 95 (2005)052002, hep-lat/0503005.

Open Access This chapter is licensed under the terms of the Creative Commons Attribution 4.0 International License (http://creativecommons.org/licenses/by/4.0/), which permits use, sharing, adaptation, distribution and reproduction in any medium or format, as long as you give appropriate credit to the original author(s) and the source, provide a link to the Creative Commons licence and indicate if changes were made.

The images or other third party material in this chapter are included in the chapter's Creative Commons licence, unless indicated otherwise in a credit line to the material. If material is not included in the chapter's Creative Commons licence and your intended use is not permitted by statutory regulation or exceeds the permitted use, you will need to obtain permission directly from the copyright holder. 